\title{
Einleitung: Affen, Menschen, Affekte
}

To know how an ape thinks and feels is to imagine its consciousness, as a novelist might imagine the interior life of a character. $^{1}$

\subsection{Ein Planet der Affen}

Affen und Affekte - diesen Bezug stellt schon ein Klassiker der Science Fiction her: Pierre Boulles Roman La planète des singes ${ }^{2}$ von 1963. Boulle hat mit seinem Titel durchaus recht: Wir leben auf einem Planeten der Affen. Der Mensch, der diesen Planeten mehr oder minder frei nach seinem Willen umzugestalten weiß, ist biologisch-taxonomisch gesehen nichts anderes als eine weitere, wenn auch biogeographisch betrachtet sehr erfolgreiche Primatenspezies. Dass der Mensch jedoch als homo sapiens von seinen nächsten Artverwandten - den pans, gorillas und pongos, gar den hylobatidae - geschieden wird (statt etwa als pan sapiens zu fungieren oder ,den Anderen “ einen homo gorilla zuzugestehen), jene aber wiederum aufgrund dieser Verwandtschaft taxonomisch als hominidae gruppiert, ist Teil einer traditionsreichen und affektiven Rhetorik der anthropologischen Differenz.

Diese Rhetorik kreist um eine alte naturgeschichtliche und anthropologische Frage, die auch das Erkenntnisinteresse jener Wissenschaft noch prägt, die sich mit der Biologie, Paläontologie, Ökologie und Ethologie der Primaten ${ }^{3}$ befasst - der

\footnotetext{
${ }^{1}$ Daston, Lorraine: Intelligences. Angelic, Animal, Human. In: Lorraine Daston/Gregg Mitman (Hg.): Thinking With Animals. New Perspectives on Anthropomorphism. New York 2005, S. 37-58, hier S. 40.

${ }^{2}$ Boulle, Pierre: La planète des singes. Paris 1963. Im Folgenden, wenn zur Unterscheidung nötig, als $P S$ geführt; Seitenangaben im Text.

${ }^{3}$ Die Problematik des Begriffs ,Primaten' für die An-Ordnung all jener Säugetiere, die mit dem Menschen am engsten verwandt sind, zeigt sich an der Schwierigkeit, die Frage „Was sind Primaten?“ zu beantworten. Siehe Geissmann, Thomas: Vergleichende Primatologie. Berlin u. a. 2003, S. 6 ff.
} 
Primatologie: Was macht den Menschen zum Menschen? ${ }^{4}$ Bei allem Interesse für die jeweils eigene Evolution, Ökologie und Charakteristik einer jeden Primatenspezies und die evolutionär bedingten Gemeinsamkeiten aller Primaten, bleibt auch die Affenforschung doch häufig dieser Frage nach einer anthropologischen Differenz verpflichtet: Was unterscheidet den homo sapiens von den anderen Affen? ${ }^{5}$

Wie Boulles Protagonist Ulysse Mérou in La planète des singes feststellen muss: nicht allzu viel. Als Teil einer wissenschaftlichen Expedition reist er in die Tiefen des Alls zu einem Sonnensystem, das dem unseren gleicht, zu einem Planeten, der so sehr der Erde ähnelt, dass man ihn ,Soror' nennt. Auch die Gesellschaft, die Ulysse und seine Begleiter antreffen, gleicht überwiegend jener der Erde: eine technologisierte Kultur aus der ersten Hälfte des 20. Jahrhunderts. Mit einem gravierenden Unterschied, wie Ulysse Mérou mit zunehmendem Grauen wahrnehmen muss: Die Rollen von Affen und Menschen sind hier verkehrt. ${ }^{6}$

Es sind drei unterschiedliche große Affenarten, die diese Welt in einem komplexen hierarchischen Gefüge beherrschen, Orangutans, Schimpansen und Gorillas, und es sind die Menschen, die nackt, sprachlos und leeren Blicks gejagt, erforscht und getötet werden. Hier kommen die Affekte ins Spiel. Für Ulysse ist die Erfahrung dieses Rollentausches eine infame Erniedrigung:

Oui! moi, un des rois de la création, je commençai à tourner en rond autour de ma belle. Moi, l'ultime chef d'œuvre d'une évolution millénaire, devant tous ces singes assemblés qui m’observaient avec avidité, devant un vieil orang-outan qui dictait des notes à sa secrétaire, devant un chimpanzé femelle qui sourirait d'un air complaisant, devant deux gorilles ricanant, moi, un homme invoquant l'excuse de circonstances cosmiques exceptionnelles, bien persuadé en cet instant qu'il existe plus de choses sur les planètes et dans le ciel que n'en a jamais rêvé la philosophie humaine, moi, Ulysse Mérou, j'entamai à la façon des paons, autour de la merveilleuse Nova, la parade de l'amour (84 f.).

Die Krone der Schöpfung, das Meisterwerk jahrtausendelanger Evolution muss vor den versammelten äffischen Forschenden in seinem Käfig im Labor wie ein Pfau ein Balzverhalten aufführen. Die gefühlte Erniedrigung des Protagonisten ist vor allem in der Beobachtungssituation begründet. Ulysse, der in dem ihm zugeordneten menschlichen Weibchen Nova immerhin ein attraktives, ihm

\footnotetext{
${ }^{4}$ Siehe Wild, Markus: Anthropologische Differenz. In: Roland Borgards (Hg.): Tiere. Kulturwissenschaftliches Handbuch. Stuttgart 2016, S. 47-59.

${ }^{5}$ So schreibt Thomas Geissmann in seiner Einführung in die vergleichende Primatologie: „Alle Organismen sind durch den Prozess der biologischen Evolution entstanden und leiten sich entsprechend von gemeinsamen Vorfahren ab. [...] Entsprechend liegt der Schlüssel zum Verständnis der phylogenetischen Herkunft und der biologischen Merkmale des Menschen im Studium der vergleichenden Primatologie (Primatenkunde)“. Geissmann: Vergleichende Primatologie, S. 3.

${ }^{6}$ Auch wenn aus biologischer und taxonomischer Sicht die begriffliche Konstruktion der Affe/ Mensch-Differenz problematisch ist, so soll im Folgenden doch im Sinne des vertrauten Sprachgebrauchs zur Unterscheidung von menschlichen und nicht-menschlichen Primaten auf das Begriffspaar Mensch und Affe zurückgegriffen werden.
} 
zugetanes Exemplar gewinnt, empfindet eine solche Beobachtungssituation als besonders peinigend, da er selbst mit Forscherblick durch diese Welt reist.

Boulle sendet in diesem dystopischen Science-Fiction-Roman einen terrestrischen Besucher als (zunächst unfreiwilligen) teilnehmenden Beobachter in die bislang unbekannte Welt Sorors: Nach der Landung auf dem Planeten rasch und unwillentlich als Mensch unter animalisierten Menschen gefangengenommen, wird Ulysse zum Versuchsobjekt in einer Forschungsstation, kann das Vertrauen der schimpansischen Forscherin Zira und ihres Verlobten Cornelius gewinnen, sein denkendes Bewusstsein mit deren Hilfe öffentlich machen und in der Folge an der Affengesellschaft teilnehmen. Ulysse beobachtet die Gegebenheiten dieser Welt und das Verhalten ihrer Bewohner/innen genau, und er versucht, aus diesen Beobachtungen Schlüsse zu ziehen, das Verhalten zu interpretieren und aus den Erkenntnissen ein Verständnis dieses Affenplaneten und seiner Geschichte zu gewinnen:

Je repassai dans ma tête toutes mes observations, souvent enregistrées à mon insu. Une impression générale les dominait toutes: ces singes, mâles et femelles, gorilles et chimpanzés, n'étaient en aucune façon ridicule. J'ai déjà mentionné qu'ils m'étaient jamais apparus comme des animaux déguisé, comme les singes savant qu'on montre dans nos cirques. Sur Terre, un chapeau sur la tête d'une guenon est un spectacle hilarant pour certains, pour moi pénible. Rien de tel ici. Le chapeau et la tête étaient en harmonie et il n'y avait rien que de très naturel dans tous leurs gestes. La guenon qui buvait dans un verre avec une paille avait l'air d'une dame. [...] J'avais eu besoin de réfléchir pour conclure au paradoxe (56 f., Hervorheb. im Original).

Ulysse will nicht nur Zeugnis ablegen von den absurden Geschehnissen, die ihn auf Soror ereilen; ${ }^{7}$ er will eine ,idée assez précise du monde simien“ (108) gewinnen und seiner Leserschaft präsentieren. Pierre Boulles Protagonist lässt sich daher als Feldforscher betrachten, der von seinem ca. einjährigen Aufenthalt in dieser verkehrten Welt möglichst fundiert zu berichten versucht: „J'avais commencé moi-même à faire quelques observations, pensant en agrémenter le reportage que je comptais publier lors demon retour sur la Terre" (82). Mehr noch: Auf Basis seiner Beobachtungen sowohl zur Affen- als auch zur menschlichen Bevölkerung des Planeten versucht Ulysse Mérou herauszufinden, wie es zu dieser Verkehrung der gewohnten Herrschaftsverhältnisse kommen konnte. ${ }^{8}$

\footnotetext{
${ }^{7}$ Sehr wohl aber will er diese Zeugenschaft instrumentalisieren: Ulysse sieht seine „mission“ (PS, S. 159) schließlich darin, seine „frêres humains“ (S. 184), denen er doch nichts anderes als ein „,erveau d'un animal“ (S. 159) bescheinigen mag, aus der Gefangenschaft und Unterdrückung zu befreien: „Mais je reviendrais. Oui, plus tard, j'en fais le serment en évoquant les prisonniers des cages, je reviendrais avec d'autres atouts" (S. 184).

${ }^{8}$ „Mon délire tourne sans fin autour de ce problème. Certes, nous autres, civilisations, nous savons depuis longtemps que nous sommes mortelles, mais une disparation aussi totale accable l'esprit. Choc brutal? Cataclysme? Ou bien lente dégradation des uns et ascension progressive des autres? Je penche pour cette dernière hypothèse et je découvre des indices extrêmement suggestifs au sujet de cette évolution, dans la condition et dans les préoccupations actuelles des singes“. PS, S. $154 \mathrm{f}$.
} 
Seine Erzählung ist ein autobiographischer Bericht, der von den Beobachtungen und Erfahrungen erzählt, die er auf dem Planeten ,im Feld' macht, und der zugleich nach evolutionären Erklärungen für das Verhalten und die Gesellschaftsstruktur der von ihm beobachteten Primaten - Affen und Menschen - sucht. La planète des singes ist also nicht nur ein fiktiver Reise- und Forschungsbericht; der Roman weist 1963 in der Fiktion auch auf ein Genre voraus, das zeitgenössisch im Zusammenhang mit der Aufnahme und Etablierung primatologischer Langzeit-Feldstudien im Entstehen ist, und das ich im Folgenden unter dem Begriff der primatologischen Forschungsmemoiren fassen möchte.

1963 ist auch das Jahr, in dem der Biologe George Schaller seine primatologische Studie The Mountain Gorilla veröffentlicht und Jane Goodalls erster Artikel im National Geographic Magazine erscheint. ${ }^{9}$ Nur wenige Jahre später veröffentlichen beide Monographien, die ihre Zeit im Feld bei den jeweiligen Affenpopulationen umfassender beschreiben, als dies der akademische Forschungsbericht oder die Bildreportagen für das Publikationsorgan der National Geographic Society als eine der wichtigsten Geldgeberinnen gewähren können: George Schallers The Year of the Gorilla erscheint 1964, Jane Goodalls In the Shadow of Man $1971 .{ }^{10}$ Die anglophon geprägte Freiland-Primatologie ist auf dem Vormarsch und gewinnt an Öffentlichkeit; die von ihr zutage geförderten Einsichten in Verhalten und Ökologie der Affen werden rasch popularisiert; die intrikate Beziehung von Forschenden und Beforschten und die außergewöhnlichen Bedingungen der Wissensproduktion ,in der Wildnis ' stoßen auch auf nicht-wissenschaftliches Interesse. ${ }^{11}$

In La planète des singes ist die Umkehrung der Verhältnisse als Voraussetzung für die teilnehmende Beobachterposition jedoch bei Weitem kein willkommener Perspektivwechsel für Ulysse. Zwar sieht er sich angesichts der Qualen, denen die Menschen auf Soror ausgesetzt sind, immer wieder mit seinem eigenen Wissen darüber konfrontiert, dass ganz Ähnliches in seiner eigenen Welt mit Affen geschieht. ${ }^{12}$ Der Roman hält darin seiner zeitgenössischen Gesellschaft einen Spiegel hinsichtlich ihres Umgangs mit ihren Schwesterspezies vor. Doch wie Ulysses Reaktionen von Verblüffung (,surprise“ und „stupéfaction“, 44; ,étonnement“, 45; „stupeur“, 46) über Grauen und Entsetzen (,cette horreur insoutenable“, „glacé de

\footnotetext{
${ }^{9}$ Schaller, George: The Mountain Gorilla. Chicago 1963; Goodall, Jane: My Life Among Wild Chimpanzees. In: National Geographic 124 (1963), S. 272-308.

${ }^{10}$ Schaller, George: The Year of the Gorilla. London 1967; van Lawick-Goodall, Jane: In The Shadow of Man. London 1971. Im Folgenden, wenn zur Unterscheidung nötig, als YG und SM geführt; Seitenangaben im Text.

${ }^{11}$ Vgl. zur „simian science as public spectacle“ Noble, Brian E.: Politics, Gender, and Worldly Primatology: The Goodall-Fossey Nexus. In: Shirley C. Strum/Linda Marie Fedigan (Hg.): Primate Encounters. Models of Science, Gender, and Society. Chicago 2000, S. 436-462, hier S. 436.

${ }^{12}$ Ulysse erwähnt die Verwendung von Affen zu Unterhaltungszwecken („les singes savants qu'on montre dans nos cirques“, PS, S. 56), in der Forschung („Dans l'ordre ancien, beaucoup des singes devaient servir de sujets d'expérience aux hommes, comme c'est le cas dans nos laboratoires“, S. 155), und für die Jagd.
} 
terreur“, 46; „Levain se mit à claquer des dents et à trembler de tous ses membres“, 47; „,e tableau [...] grotesque et [...] diabolique“, 51) bis hin zu hysterischer Albernheit zeigen, ist diese Umkehrung - bereits zu Beginn zugespitzt in einer Jagdszene (40 ff.) - nicht allein eine hochaffektive Angelegenheit. Sie wird als verstörende Ordnungsverletzung wahrgenommen, die sich bei Ulysse symptomatisch als emotionaler Ausbruch äußert:

Il y avait dans cette scène une horreur disproportionnée avec la résistance d'un cerveau normal. [...] Et mon émoi explosa encore d'une manière absurde, en harmonie avec le côté grotesque de cette macabre exposition. Je me laissai aller à une hilarité insensée; j'éclatai de rire (53, Hervorheb. MS).

Es ist nicht irgendein Tier, das hier den Menschen als Anführer der belebten Welt ersetzt hat. Es sind Affen, mit einer ,physique totalement, absolument simies$q u e^{\text {" }}$ (52, Hervorheb. im Original) aber ,par-dessus tout le caractère humain [...] [d'] expression“" (45). Ausgerechnet die Versuchstiere und Hofnarren der Menschen; die ,garstigen Affen', die als Vergleich für den Menschen herangezogen als Beleidigung dienen, ${ }^{13}$ die evolutionären Schattengeschwister der menschlichen Phylogenese, ausgerechnet die zum Veröffentlichungszeitpunkt des Romans gerade erst seit ein paar Jahren im Feld beforschten Affen haben die Herrschaft über den Planeten übernommen. Wie sich an Ulysses Reaktion auf diesen Planeten der Affen zeigt, nimmt der Affe auf unserem Planeten eine besondere Funktion für das Selbstverständnis des Menschen ein: Als Distinktionsfigur ist der Affe von ambivalenter emotionaler Bedeutung.

Indem Pierre Boulles Roman die Charakteristika eines Feldforschungsaufenthaltes ,unter Affen“ und des darauf basierenden autobiographischen Berichtens von der Reise- und Forschungserfahrung mit dem affektiv ambivalenten Verhältnis kombiniert, welches der Mensch zum Affen pflegt, kündet der Roman nicht nur von der weitreichenden kulturellen Faszination für die Primatologie und das durch sie erweiterte Wissen auf der Spurensuche zur Entstehung des Menschen sowie für ihre wissenschaftlichen Praktiken und Figuren. Er illustriert auch die Fragen, die den Anlass für die vorliegende Arbeit geben: Welche Rolle spielt Emotionalität für unser Verhältnis zu anderen Primaten? Wie steuert, was wir fühlen unsere Wahrnehmung, unser Wissen und dessen Vermittlung und Darstellung? Wie entsteht Erkenntnis ,im Feld'? Welchen Platz nehmen Affekte, Gefühle und Emotionen in den Praktiken, in der Wissensproduktion und der Vermittlung von Forschung ein? Was sind spezifische Affekte des Forschens mit Affen, wie äußern sie sich, wozu dienen sie, wie werden sie dargestellt und thematisiert, worin sind sie eingebettet, womit hängen sie zusammen, und wer berichtet wie und wann von ihnen?

\footnotetext{
${ }^{13}$ Vgl. Shah, Mira: Garstige Affinitäten. Frauen und Affen in J.W. Goethes ,Die Wahlverwandtschaften‘. In: Orbis Litterarum 70/2 (2015), S. 108-149.
} 


\subsection{Die Affekte der Forschenden}

Diese ersten Leitfragen für die vorliegende Forschungsarbeit schließen an den Forschungsstand der Wissenschafts- und Emotionsforschung an. Sie ergeben sich aber vor allem aus dem Material dieser Studie selbst. 1990 erscheinen Jane Goodalls zweite Forschungsmemoiren unter dem Titel Through a Window. ${ }^{14}$ Dieser Titel bezieht sich auf eine Metapher, die Goodall ihrem Erkenntnisverfahren zugrunde legt:

There are many windows through which we can look into the world, searching for meaning. There are those opened up by science, their panes polished by a succession of brilliant, penetrating minds. Through these we can see even further, even more clearly, into areas that once lay beyond human knowledge. Gazing through such a window I have, over the years, learned much about chimpanzee behaviour and their place in the nature of things. And this, in turn, has helped us to understand a little better some aspects of human behaviour, our own place in nature (8).

Es gibt jedoch nicht nur diese Fenster szientistischer Klarsicht, fährt Goodall fort, denn:

[T] here are other windows; windows that have been unshuttered by the logic of philosophers; windows through which the mystics seek their visions of the truth; windows from which the leaders of great religions have peered as they searched for purpose not only in the wondrous beauty of the world, but also in its darkness and ugliness. Most of us, when we ponder on the mystery of our existence, peer through but one of these windows onto the world. And even that one is often misted over by the breath of our finite humanity. We clear a tiny peephole and stare through (8).

Goodalls philosophische, mystische und religiöse Fenster, die jeweils ihren eigenen, teils opaken Blick auf, die Welt ${ }^{6} \mathrm{zu}$ bieten versprechen, symbolisieren Zugänge zur Erschließung von Wissen. Einen weiteren lässt Goodall merkwürdigerweise aus - vielleicht, weil er kaum zu trennen ist von all den anderen Fenstern und eher der Wirkung des ,breath of our finite humanity“ nahekommt: Emotionalität. ${ }^{15}$ Denn so unterschiedlich die Zugänge der Emotionsforschung zu

\footnotetext{
${ }^{14}$ Goodall, Jane: Through A Window. 30 Years with the Chimpanzees of Gombe, London 1990. Im Folgenden, wenn zur Unterscheidung nötig, als TW geführt; Seitenangaben im Text.

${ }^{15} \mathrm{Um}$ dem Minenfeld begriffsgeschichtlicher, emotionstheoretischer, philosophischer oder soziologischer Feindifferenzierungen zu entgehen und einen offenen Blick auf alle emotionalen Phänomene beibehalten zu können, soll im Folgenden nicht der Versuch unternommen werden, Affekte, Emotionen, Leidenschaften, Gefühle, Stimmungen etc. definitorisch voneinander zu scheiden. Vielmehr dient in Anlehnung an Katrin Pahls Vorschlag der Begriff Emotionalität als Leitkategorie. Mit diesem Begriff fasst Pahl das häufig latente aber auch ausgeübte Vermögen, emotional zu sein. Emotionen sind dabei spezifische Manifestationen von Emotionalität. Mit dem Begriff als übergreifende Kategorie kann sowohl die Komplexität (etwa ihre ,Vermengtheit" und Wandelbarkeit) emotionaler Phänomene als auch ihre ,doppelte Modalität', zugleich ,reale' körperlich-psychische Phänomene und kulturell, sozial und politisch geformt zu sein,
} 
ihrem Gegenstand sind, ${ }^{16}$ sie einigen sich doch darauf, dass die Fähigkeit zur und Notwendigkeit von Emotionalität eine anthropologische Konstante ist. ${ }^{17}$

Dass Goodall in ihrer Reflexion verschiedener Zugangsweisen zu einem Verständnis von Welt nicht von Emotionalität spricht - in ihren Forschungsmemoiren zugleich aber, wie im Folgenden im Einzelnen analysieren wird, häufig ihr affektives Empfinden thematisiert -, liegt jedoch auch daran, dass Emotionalität einen schweren Stand hat. In der wissenschaftlichen Forschung sind Emotionen prekär: Als Teil eines übergreifenden Paradigmas wissenschaftlicher Objektivität wird Emotionalität gerade (aber nicht nur) in den Naturwissenschaften überwiegend als geächtetes Neben- und Ausschussprodukt problematischer Subjektivität in Forschungsprozessen betrachtet. Die Grundlage einer solchen Auffassung von Objektivität vs. Subjektivität findet sich in der traditionsreichen Konzeptualisierung eines Widerstreits zwischen Rationalität und Emotionalität, die meist an einer cartesianischen Vernunft/Gefühl-Dichotomie festgemacht wird, aber weit über diese hinaus in die europäische Antike zurückreicht. ${ }^{18}$ Das Konzept

berücksichtigt werden. Zudem suggeriert der Begriff bei Pahl eine immer schon anzunehmende Überlappung von Rationalität und Emotionalität, die für die Frage nach der epistemologischen Rolle von Emotionalität ausschlaggebend ist. Vgl. Pahl, Katrin: Emotionality: A Brief Introduction. In: Modern Language Notes 124/3 (2009), S. 547-554; Pahl, Katrin: Tropes of Transport. Hegel and Emotion. Evanston 2012, S. 5. ,Emotion', ,Affekt' und ,Gefühl“ werden im Folgenden, sofern nicht anders ausgewiesen, synonym verwendet. Zur Vielfalt und Geschichte der Begriffe siehe jedoch Frevert, Ute u. a. (Hg.): Gefühlswissen. Eine lexikalische Spurensuche in der Moderne. Frankfurt am Main/New York 2011; Engelen, Eva-Marie: Emotion als wissenschaftliche Kategorie. In: Rainer Maria Kiesow/Martin Korte (Hg.): EGB. Emotionales Gesetzbuch. Dekalog der Gefühle. Köln/Weimar/Wien 2005, S. 289-307.

${ }^{16}$ Vgl. Newmark, Catherine: Passion - Affekt - Gefühl. Philosophische Theorien der Emotionen zwischen Aristoteles und Kant. Hamburg 2008; von Koppenfels, Martin/Zumbusch, Cornelia: Einleitung. In: Dies. (Hg.): Handbuch Literatur \& Emotionen. Berlin/Boston 2016, S. 1-36. Zur Geschichte der Emotionsforschung siehe Plamper, Jan: Geschichte und Gefühl. Grundlagen der Emotionsgeschichte. München 2012.

${ }^{17}$ So weist das Lexikon der Ästhetischen Grundbegriffe ,Affekt‘ als genuin anthropologischen Begriff aus. Emotionalität fungiert jedoch nicht unbedingt als Differenzkriterium Mensch vs. Tierreich, sondern verweist im Gegenteil auf die ,ins Tierreich zurückgehenden natürlichen Gattungseigenschaften“ des Menschen. Grimm, Hartmut: Affekt. In: Karlheinz Barck u. a. (Hg.): Ästhetische Grundbegriffe. Bd. 1. Stuttgart/Weimar 2000, S. 16-48, hier S. 18. Siehe auch Darwin, Charles: The Expressions of The Emotions in Man and Animals. London 1872. Monique Scheer untersucht allerdings Emotionen als praxeologisches Engagement mit der Welt, das beim Menschen anderen Variablen unterliegt als bei nicht-menschlichen Tieren: „Conceiving emotions as practices means understanding them as emerging from bodily dispositions conditioned by a social context, which always has cultural and historical specificity“. Scheer, Monique: Are Emotions a Kind of Practice (And Is That What Makes Them Have a History)? A Bourdieuian Approach to Understanding Emotion. In: History and Theory 51 (2012), S. 193-220, hier S. 193.

${ }^{18}$ Descartes' psychophysische Doppelbestimmung von Emotionen vor dem Hintergrund eines Geist-Körper/Leib-Seele-Dualismus in Les passions de l'âme (1649) fügt der bereits aus der Antike bekannten ethischen Abwertung der Gefühle eine normative oder epistemische hinzu: "Geht es der ethischen Abwertung darum, den motivationalen Einfluss der Gefühle auf menschliches Verhalten so gering wie möglich zu halten, so bezweifelt die normative Abwertung, dass 
einer um alles Affektive und Subjektive bereinigten wissenschaftlichen Objektivität als Norm und Ethos der Wissensproduktion ist jedoch jüngeren Datums. Im Verständnis einer solchen wissenschaftlichen Objektivität objektiv zu sein, so arbeiten Lorraine Daston und Peter Galison in ihrer wissenschaftshistorischen Studie heraus, ,is to aspire to knowledge that bears no trace of the knower - knowledge unmarked by prejudice or skill, fantasy or judgment, wishing or striving. "19 Als Konzept steht diese Mitte des 19. Jahrhunderts geformte Auffassung wissenschaftlicher Objektivität eng in Verbindung mit der Entstehung einer spezifischen Subjektivität des fühlenden Individuums um $1800 .^{20}$ Aus der cartesianischen Weltsicht des 18. Jahrhunderts resultiert für den Emotionssoziologen Jack Barbalet parallel zur Formierung einer solchen Subjektivität die Suche nach ,unpersönlicher" wissenschaftlicher Erkenntnis, zumindest aber nach Möglichkeiten, Wissenschaft als nicht-subjektiv, ergo objektiv darzustellen - „typically achieved by characterizing science in terms of its formal properties, especially those associated with its methods, rather than in terms of its human attributes, including the scientist's emotions““. ${ }^{21}$ Subjektivität muss, so zeigt sich, zunächst erst einmal als problematisch begriffen und mit einem veränderten Ziel wissenschaftlicher Forschung zusammengebracht werden, damit das neue Paradigma sich entfalten kann: Naturforscher sind nun nicht mehr auf der Suche nach der idealen Repräsentation eines Archetyps von ,Natur', ${ }^{22}$ sondern wollen ein ,reales ', ungefiltertes und unbeeinflusstes Abbild natürlicher Phänomene bieten. Folglich sehen sie sich selbst als größtes Hindernis für Erkenntnisprozesse, weil sie ihr subjektives Selbst zunehmend als störendes Element wahrnehmen: „[M]en of science began to fret openly about a new kind of obstacle to knowledge: themselves. Their fear was that the subjective self was prone to prettify, idealize, and, in the worst case,

Gefühle begründet oder unbegründet sein können. In dem Maße, wie Gefühle [...] körperlichen Empfindungen parallelisiert werden, bleiben sie arationale Phänomene, die als solche nicht normativ zugänglich sind“. Hartmann, Martin: Gefühle. Wie die Wissenschaften sie erklären. Frankfurt a. M./New York 2005, S. 15. Siehe auch Voss, Christiane: Die narrative Transformation aristotelischer und moderner Emotionstheorien. In: Martin Harbsmeier/Sebastian Möckel (Hg.): Pathos, Affekt, Emotion. Transformationen der Antike. Frankfurt a. M. 2009, S. 107-130, hier S. 108; Landweer, Hilge/Newmark, Catherine: Seelenruhe oder Langeweile, Tiefe der Gefühle oder bedrohliche Exzesse? Zur Rhetorik von Emotionsdebatten. In: Martin Harbsmeier/Sebastian Möckel (Hg.): Pathos, Affekt, Emotion. Transformationen der Antike. Frankfurt a. M. 2009, S. 79-106; Daston, Lorraine: The Moral Economy of Science. In: Osiris 10 (1995), S. 2-24.

${ }^{19}$ Daston, Lorraine/Galison, Peter: Objectivity. New York 2007, S. 17.

${ }^{20} \mathrm{Vgl}$. Frevert, Ute: Gefühlswissen in der Moderne - Entwicklungen und Ergebnisse. In: Dies. u. a. (Hg.): Gefühlswissen. Eine lexikalische Spurensuche in der Moderne. Frankfurt a. M./New York 2011, S. 263-278.

${ }^{21}$ Barbalet, Jack: Consciousness, Emotions, and Science. In: Debra Hopkins/Jochen Kleres/ Helena Flam/Helmut Kuzmics (Hg.): Theorizing Emotions. Sociological Explorations and Applications. Frankfurt a. M./New York 2009, S. 39-71, hier S. 44.

${ }^{22} \mathrm{Vgl}$. Daston/Galison: Objectivity, S. 18. 
regularize observations to fit theoretical expectations: to see what it hoped to see“. ${ }^{23}$ Das neue Ideal der Naturalisten bedingt die Selbstdisziplinierung der Forschenden: die Unterdrückung bestimmter, als subjektiv abgeschriebener Aspekte dieses Selbsts, die Einhegung von Subjektivität durch wissenschaftliche Praktiken, die sich als Technologien des Selbst lesen lassen. ${ }^{24}$ Dadurch wurde der epistemologische Anteil von Emotionalität - wie er sich bspw. in der Faszination für Wunder und das Staunen als Modus wissenschaftlicher Wahrnehmung im Rahmen der ,Scientific Revolution“ und als Teil des Diskurses der Entdeckung des (absolut) Neuen in der Frühen Neuzeit gezeigt hatte ${ }^{25}$ - nicht nur verdrängt, sondern geradezu verneint. ${ }^{26}$

Es ist bezeichnenderweise die Wiederentdeckung der klassischen Rhetorik (vor allem Aristoteles' Rhetorik) im 17. Jahrhundert, die Emotionalität in der Neuzeit zuallererst mit einem Verdacht der ,Unwissenschaftlichkeit' belegt: Nicht die ,Passionen“ in der Wissenschaft schienen zunächst problematisch, ,but the dangers of passion in persuasion, a fear of distortion in scientific communication through emotion in eloquence“".27 Dieser von Barbalet hergestellte Bezug zwischen den epistemologischen Praktiken der Naturforschung und -philosophie und der Rolle von Emotionen in der Rhetorik wissenschaftlicher Kommunikation erhellt auch die Übertragung eines Reinigungs-Gedankens auf die Idee einer fortschreitenden ,Zivilisierung ' des Menschen als Geschichte der Moderne. ${ }^{28}$ Zentral

\footnotetext{
${ }^{23}$ Daston/Galison: Objectivity, S. 34.

${ }^{24}$ Vgl. Daston/Galison: Objectivity, S. 39; Foucault, Michel: Technologien des Selbst. In: Ders.: Ästhetik der Existenz. Schriften zur Lebenskunst. Frankfurt a. M 2007 (frz. 1994), S. 287-317.

${ }^{25}$ Vgl. Greenblatt, Stephen: Marvelous Possessions. The Wonder of the New World. Chicago/ Oxford 1991, S. 20. Daston/Park sprechen von „,a history of wonder as a passion of natural inquiry“ als "history of the evolving collective sensiblities of naturalists“, die ihrerseits zeige, wie objektive Ordnung und subjektive Wahrnehmung sich nicht gegenseitig ausschließen, sondern schlicht zwei Seiten einer Medaille seien. Daston, Lorraine/Park, Katherine: Wonder and the Order of Nature 1150-1750. New York 1998, S. 14.

${ }^{26}$ Barbalet weist aber darauf hin, dass diese Selbstwahrnehmung der wissenschaftlichen Gemeinschaft zweierlei emotionale Aspekte nicht ausschließe, sondern geradezu affirmativ propagiere: „the motivation of the scientist's investigation and commitment of the scientist to the social institution of science“; Emotionen sind somit „,confined to the motivational framework of science and not its interior". Barbalet: Consciousness, S. 45 f., Hervorheb. MS.

${ }^{27}$ Barbalet: Consciousness, Hervorheb. MS.

${ }^{28}$ Siehe Elias, Norbert: Über den Prozess der Zivilisation. Soziogenetische und psychogenetische Untersuchungen [1939]. Frankfurt a. M. 1997. Elias' Programm beschreibe eine zunehmende Affektkontrolle als zentrales Element bei der Herausbildung und Stabilisierung moderner Gesellschaften (aber eben auch bei der Festigung wissenschaftlicher Paradigmen), so Becker, Patrick: Rationalisierung des Gefühls - Emotionalisierung der Vernunft. Zum Umgang mit Emotionen in der Gegenwartsgesellschaft. In: Moderne. Kulturwissenschaftliches Jahrbuch 3 (2007), S. 63-78, hier S. 66. Zu einer Kritik von Elias unter Berücksichtigung seines Affektbegriffs siehe Frevert, Ute: Gefühle definieren: Begriffe und Debatten aus drei Jahrhunderten. In: Dies. u. a. (Hg.): Gefühlswissen. Eine lexikalische Spurensuche in der Moderne, Frankfurt a. M./New York 2011, S. 9-39, hier S. $11 \mathrm{ff}$.
} 
für die sich selbst u. a. durch ihre wissenschaftliche Professionalisierung definierende Moderne ist die Vorstellung einer notwendigen und erfolgreichen Reinigung des Menschen an sich, allen voran aber des ,homo scientificus ' von seinen nicht-objektivierbaren Einflüssen und Charakteristika, besonders seinen subjektiven, verwirrenden, beeinträchtigenden, oder anderweitig , unwissenschaftlichen * Emotionen. ${ }^{29}$ Der zwielichtige Ruf der Emotionen wandert aus der (antiken) Rhetorik über die Medien ihrer Vermittlung in die Praktiken der Wissenschaft selbst hinein. Gepaart mit einer weiteren berühmten Entleihung aus der Antike - einem Katharsis-Verständnis, das die Reinigung von (problematischer) Emotionalität impliziert - wird so ein weitreichendes Selbstverständnis der Moderne als ,rational' (und nicht emotional) ausgeprägt. ${ }^{30}$

Weder aus phänomenologischer noch aus epistemologischer Warte ist diese pejorative Sicht auf Emotionalität und die Forderung nach der Läuterung des forschenden Subjektes von seiner Subjektivität im Namen wissenschaftlicher Objektivität aufrechtzuerhalten. Bereits Ludwik Flecks vergleichende Erkenntnistheorie stellt 1935 diese Scheidungen von Rationalität und Emotionalität, Objektivität und Subjektivität, ja selbst Objekt und Subjekt infrage: Erkenntnis müsse, so Fleck, als Ergebnis sozialer Tätigkeit untersucht werden, die als solche unweigerlich mit emotionalen Komponenten einhergehe. ${ }^{31}$ Michael Polanyi fügt dieser sozialen Einbettung von Erkenntnis in seiner Objektivismus-Kritik ein Schlaglicht auf den jedem Erkennen zwangsläufig inhärenten Anthropozentrismus bei. Den Anspruch auf Objektivität im Sinne eines ,abandon [of] all sentimental egoism, and to see ourselves objectively in the true perspective of time and

\footnotetext{
${ }^{29}$ Vgl. Rosenwein, Barbara H.: Worrying about Emotions in History. In: The American Historical Review 107/3 (2002), S. 821-845, hier S. 826 ff. Landweer/Newmark haben ihrerseits darauf hingewiesen, dass die Emotionstheorie selbst rhetorisch formiert ist: Die thematische Auseinandersetzung mit Emotionen unterliegt Konjunkturen, und diese „Gefühlskonjunkturen sind spezifische rhetorische Situationen, die auf historische Gegebenheiten und tradierte Debatten reagieren; die Auffassung dessen, was Emotionen sind, ebenso wie ihre positive oder negative Einschätzung müssen als kontextabhängig in einem sehr starken Sinn verstanden werden“. Auch gelte es zu berücksichtigen, dass es in all den von ihnen angesprochenen Emotionsdebatten kaum um pauschale Befürwortung oder Ablehnung von Emotionen, sondern um „Fragen des Maßes und der Angemessenheit, um erlaubte Intensitäten, um gefährliche und ungefährliche Arten, um Status und Wert einzelner Gefühle, um sensible Rationalitäten“ gehe. Landweer/Newmark: Zur Rhetorik von Emotionsdebatten, S. $81 \mathrm{f}$.

${ }^{30} \mathrm{Zu}$ einer Problematisierung derart einengender ,moderner' Entlehnungen aus Aristoteles> komplexem Emotionsbegriffen in Rhetorik, Poetik und Politik siehe jedoch Schmitt, Arbogast: Kommentar. In: Aristoteles: Poetik. Berlin 22011, S. 193-742, hier S. 334. Zur Frage des Katharsis-Verständnisses siehe auch Abschn. 3.5.

${ }^{31} \mathrm{Vgl}$. Fleck, Ludwik: Entstehung und Entwicklung einer wissenschaftlichen Tatsache. Einführung in die Lehre vom Denkstil und Denkkollektiv [1935]. Frankfurt a. M. 1980, S. 53 f. Siehe dazu auch Jensen, Uffa: Across Different Cultures? Emotions in Science during the Early Twentieth Century. In: Frank Biess/Daniel M. Gross (Hg.): Science and Emotion After 1945. A Transatlantic Perspective. Chicago/London 2014, S. 263-277, hier S. 264; Endert, Elke: Über Zusammenhänge von Fühlen und Denken in Wahrnehmungs- und Wissensprozessen. Ein Vergleich der "Affektlogik" von Luc Ciompi mit dem wissenssoziologischen Ansatz Ludwik Flecks. Köln 1997, S. 99 f.; Daston: The Moral Economy of Science, S. 4 f.
} 
space“, 32 beschreibt Polanyi als von vornherein vergeblich, weil er die spezifisch menschliche Perspektive zu leugnen versucht:

For, as human beings, we must inevitably see the universe from a centre lying within ourselves and speak about it in terms of a human language shaped by the exigencies of human intercourse. Any attempt rigorously to eliminate our human perspective from our picture of the world must lead to absurdity. ${ }^{33}$

Teil dieser menschlichen Perspektive sind bei Polanyi „,[i]ntellectual“ oder ,scientific passions“ ${ }^{34}$ die eine heuristische Funktion für wissenschaftliche Forschung haben.

In jüngerer Zeit ist im Rahmen des ,Emotional Turn“ in den Neurowissenschaften ${ }^{35}$ bzw. des ,Affective Turn' in den zunehmend durch die neurowissenschaftliche Emotionsforschung inspirierten Geistes- und Sozialwissenschaften ${ }^{36}$ verschiedentlich nicht nur Kritik an der anhaltenden Verwendung der cartesianischen Dichotomie geübt worden; es sind auch einzelne Emotionen als Objekte der Forschung in den Fokus getreten. Dabei wird zunehmend die epistemologische Funktion von Emotionalität diskutiert und - mit Bezugnahme auf Fleck und Polanyi - die Rückkehr zur Anerkennung von emotionalen Anteilen und Bedingungen menschlicher Erkenntnis und somit auch zur Reflexion wissenschaftlicher Dogmen und Paradigmen gefordert. ${ }^{37}$ Gerade auch im wachsenden

\footnotetext{
${ }^{32}$ Polanyi, Michael: Personal Knowledge. Towards a Post-Critical Philosophy [1958]. London 1998, S. 3.

${ }^{33}$ Polanyi: Personal Knowledge, S. 3. Zum epistemologischen Anthropozentrismus-Problem siehe auch Steiner, Gary: Anthropozentrismus. In: Arianna Ferrari/Klaus Petrus (Hg.): Lexikon der Tier-Mensch-Beziehungen. Bielefeld 2015, S. 28-32.
}

${ }^{34}$ Polanyi: Personal Knowledge, S. 143.

${ }^{35} \mathrm{Biess} /$ Gross sehen eine solche Absage an das moderne Paradigma, ,that prioritized rationality and pathologized emotion“, mit der Publikation von António Damásios Descartes' Error 1994 und Daniel Golemans Emotional Intelligence 1995 beginnen. Biess, Frank/Daniel M. Gross: Emotional Returns. In: Dies.: (Hg.): Science and Emotions after 1945. A Transatlantic Perspective. Chicago/London 2014, S. 1-38, hier S. 9.

${ }^{36} \mathrm{Vgl}$. Clough, Patricia Ticineto/Halley, Jean (Hg.): The Affective Turn. Theorizing The Social. Durham/London 2007. Zu einer Kritik des diesem Turn zugrunde liegenden Affektverständnisses: Leys, Ruth: The Turn to Affect: A Critique. In: Critical Inquiry 37 (2011), S. 434-472.

${ }^{37}$ Landweer/Newmark weisen aber darauf hin, inwiefern erstens die neueren philosophischen Emotionsdebatten auf den ersten Blick stark auf die Kontinuität einer Vernunft/Gefühl-Dichotomie bezogen scheinen, die zwar historisch begründet werde, sich jedoch philosophiehistorisch nicht halten lasse. Zweitens stellen sie die Aufmerksamkeit für Emotionen in den Kontext eines neuen (in Abschn. 4.2 diskutierten) Paradigmas, insofern sie vor allem ökonomischen Zwecken, wie etwa dem Funktionieren der Menschen in der Arbeitswelt zu dienen scheint: „Daß Rationalität mit Rationalisierung, ökonomischem Nutzen und Profitmaximierung zusammenhängt, stellt keine besonders neue Entdeckung in der Philosophiegeschichte dar. Auch daß Emotionen auf ihre Nützlichkeit und Schädlichkeit hin betrachtet werden - sei es für vitale Prozesse, sei es für das rational Gewünschte -, ist ein philosophiehistorisch gängiger Topos. Daß die Emotionen aber in dieser Weise explizit und direkt dem ökonomischen Nutzenkalkül unterworfen werden, scheint doch eine spezifische Ausformung der Gegenwart zu sein“. Landweer/Newmark: Zur Rhetorik von Emotionsdebatten, S. 101. 
Bereich der Wissenschaftsgeschichte und -forschung rückte ein Interesse an Emotionen in der Wissenschaft in den letzten beiden Dekaden vermehrt ins Zentrum der Beschäftigung mit den vielfältigen Bedingungen wissenschaftlicher Praxis und Erkenntnis. ${ }^{38}$

Auch innerhalb einzelner Disziplinen hat die teilweise von der Wissenschaftsforschung und/oder von postmoderner Theoriebildung angeregte Auseinandersetzung mit Methoden, strukturellen Bedingungen und historischen Paradigmen der jeweiligen Wissensproduktion zu einer Berücksichtigung von Emotionalität geführt. Besonders deutlich zeigt sich dies am literaturwissenschaftlich informierten ,Reflexive Turn ${ }^{639}$ in der Ethnologie. ${ }^{40}$ Während die Theorie-Eingaben der Moderne und Postmoderne sich jedoch in der Ethnologie vor allem an Fragen nach der, literarischen Gemachtheit' ethnologischen Wissens, also den hermeneutischen und narrativen Grundverfahren des Studiums von Kultur und Gesellschaft ablagern ${ }^{41}$ und zu fachinternen, fachextern informierten Debatten führen, bleibt die Beschäftigung mit

\footnotetext{
${ }^{38}$ Siehe Latour/Woolgars Untersuchung der wissenschaftlichen Konstruktion von Fakten im interrelationalen Umfeld des Labors, Fox Kellers Analysen des Konnex von wissenschaftlichem Objektivitäts- und Männlichkeitsideal sowie Dastons Arbeiten zur moralischen Ökonomie und der Genese des Objektivitätsideals sowie zum Anthropomorphismus: Latour, Bruno/Woolgar, Steve: Laboratory Life. The Social Construction of Scientific Facts. Princeton ${ }^{2}$ 1986; Fox Keller, Evelyn: A Feeling for the Organism: The Life and Work of Barbara McClintock. San Francisco 1983; Fox Keller, Evelyn: Reflections on Gender and Science. New Haven/London 1995; Daston: The Moral Economy of Science; Daston/Galison: Objectivity; Daston: Intelligences. Siehe auch Bauer, Susanne/Heinemann, Torsten/Lemke, Thomas: Einleitung. In: Dies. (Hg.): Science and Technology Studies. Klassische Positionen und aktuelle Perspektiven. Berlin 2017, S. 7-40.

${ }^{39}$ Zur Einbettung des ,Reflexive Turn' in breitere postmoderne ,Turns' siehe Bachmann-Medick, Doris: Cultural Turns. Neuorientierungen in den Kulturwissenschaften. Reinbek 2014, S. 144 ff. Zur Nähe und Verknüpfung von Literaturwissenschaft und Anthropologie/Ethnologie in der Reflexion von Repräsentationsformen siehe Fuchs, Martin/Berg, Eberhard: Phänomenologie der Differenz. Reflexionsstufen ethnographischer Repräsentation. In: Dies. (Hg.): Kultur, soziale Praxis, Text. Die Krise der ethnographischen Repräsentation. Frankfurt a. M. 1993, S. 11-108.

${ }^{40} \mathrm{Im}$ Englischen wird ein Großteil der anthropologischen Sparte, die im Deutschen als Ethnologie fungiert, mittlerweile mit dem Begriff , social anthropology ${ }^{\star}$ bezeichnet, der im deutschen Sprachgebrauch historisch-ideologisch vorbelastet scheint. Siehe Sigrist, Christian: Sozialanthropologie; Social anthropology. In: Karlfried Gründer (Hg.): Historisches Wörterbuch der Philosophie. Bd. 9. Basel: Schwabe 1995, S. 1121-1126; Kohl, Karl-Heinz: Ethnologie - die Wissenschaft vom kulturell Fremden. Eine Einführung. München 32012, S. 14. Ich verwende den zunächst nicht zwischen Sozial- und Kulturanthropologie differenzierenden deutschen Begriff Ethnologie, es sei denn, es ist durch die Quellen anders indiziert.

${ }^{41}$ So sieht Clifford Geertz ein, dass ,,what we call our data are really our own constructions of other people's constructions of what they and their compatriots are up to“, und schlägt vor, Kultur als Text zu lesen. Geertz, Clifford: Thick Description: Toward an Interpretative Theory of Culture. In: Ders.: The Interpretation of Cultures. New York 1973, S. 3-30, hier S. 9. James Clifford verwendet sogar den Begriff Fiktion, um nicht nur die ,partiality of cultural and historical truths, the way they are systematic and exclusive", zu beschreiben, sondern wie schon Geertz auch auf den Charakter ethnographischen Wissens als Produkt von Imagination hinzuweisen. Clifford, James: Introduction: Partial Truths. In: James Clifford/George E. Marcus (Hg.): Writing Culture. The Poetics and Politics of Ethnography. Berkeley u. a. 1986, S. 1-26, hier S. 6.
} 
Emotionalität als Teil von Forschungs- und Erkenntnisprozessen zunächst noch in einer neuen Aufmerksamkeit für Subjektivität in der ethnologischen Forschungspraxis und Kulturrepräsentation verborgen. Sensibilisiert durch ihren teilweise prekären Status reflektieren aber vor allem weibliche Forschende in den 1960er und 1970er Jahren ihre persönlichen Umstände im Feld und wie diese die Beziehungen zu Informant/innen, das Zusammentragen und Interpretieren von Daten und die Befähigung zur Forschungsarbeit bestimmen. ${ }^{42}$ Der Fokus liegt dabei zunächst nicht auf Emotionalität, sondern aufgrund der Idee, ,that the anthropologist can learn significant lessons about her own data by reflecting on her personal role in the field“, ${ }^{43}$ auf methodischen Fragen danach, wie sich Selbst-Reflexivität in der Feldforschung implementieren lässt und welche Rolle Geschlecht in sozialen Systemen spielt. Emotionalität wird dabei zwar als Phänomen registriert, jedoch überwiegend im Sinne des Paradigmas wissenschaftlicher Objektivität noch als eine auszuweisende und zu beherrschende Problematik des Individuums betrachtet. Im Zuge der postmodernistischen Writing-Culture-Debatte in den 1980er Jahren werden im Generationenwechsel und in der interdisziplinären Öffnung der Ethnologie (besonders hin $\mathrm{zu}$ den Gender und Postcolonial Studies) ${ }^{44}$ aus Fragen der Interpretation (Geertz, Golde) Fragen der Repräsentation (Clifford/Marcus). ${ }^{45}$ Aus der Frage nach der Positionalität ${ }^{46}$ der Forschenden wird die Frage nach der Situationalität des Wissens selbst. ${ }^{47}$ Subkutan wird das ,Emotionalitäts-Problem ' dabei dennoch verhandelt: Denn ,the peculiar emotional state we anthropologists developed when we were working in the field" ". ${ }^{48}$ wie Golde es nennt, macht sich zunehmend textuell bemerkbar. Seit Mitte des 20. Jahrhunderts erscheinen immer mehr Texte, die auf die eine oder andere Weise Emotionalität in der Anthropologie verhandeln. Der Aufsehen erregenden Veröffentlichung von Bronislaw Malinowskis Diary

\footnotetext{
${ }^{42}$ Vgl. Eriksen, Thomas Hylland/Nielsen, Finn Sivert: A History of Anthropology. London 2013, S. 152 ff.; Golde, Peggy: Introduction. In: Dies. (Hg.): Women in the Field. Anthropological Experiences. Berkeley u. a. 1986.

${ }^{43}$ Eriksen/Nielsen: History of Anthropology, S. 153.

${ }^{44} \mathrm{Vgl}$. Lubrich, Oliver/Stodulka, Thomas/Liebal, Katja: Affekte im Feld - Ein blinder Fleck der Forschung? In: Gerald Hartung/Matthias Herrgen (Hg.): Interdisziplinäre Anthropologie. Wiesbaden 2018, S. 179-197, hier S. $180 \mathrm{ff}$.

${ }^{45} \mathrm{Vgl}$. Eriksen/Nielsen: History of Anthropology, S. $180 \mathrm{ff}$.

${ }^{46}$ „Positionality is the practice of a researcher delineating his or her own position in relation to the study, with the implication that this position may influence aspect [sic] of the study, such as the data collected or the way in which it is interpreted". Qin, Donxiao: Positionality. In: Nancy Naples (Hg.): The Wiley Blackwell Encyclopedia of Gender and Sexuality Studies. Malden 2016, https:// onlinelibrary.wiley.com/doi/full/10.1002/9781118663.219.wbegss619 (12.05.2018).

${ }^{47}$, ,All knowledge was situated, and more often than not it served to justify existing power structures“. Eriksen/Nielsen: History of Anthropology, S. 174, Hervorheb. im Original. Zum Versuch einer feministischen Neuformierung von Objektivität als ,positioned rationality“ siehe Haraway, Donna: Situated Knowledges: The Science Question in Feminism and the Privilege of Partial Perspective. In: Feminist Studies 14 (Autumn 1988), S. 575-599, hier S. 590.
}

${ }^{48}$ Golde: Introduction, S. 11. 
gesellen sich bspw. der unter Pseudonym geschriebene , anthropologische Roman Return to Laughter von Laura Bohannan (1954) und Hortense Powdermakers Stranger and Friend (1966) bei. ${ }^{49}$ Derart ,persönlichen' Narrativen schlägt die Kritik entgegen, es handele sich bei ihnen um eine unwissenschaftliche und sentimentale Nabelschau. ${ }^{50}$ Die von ihren Emotionen Schreibenden kontern mit dem Verweis auf den beispielhaften Effekt eines Sprechens und Schreibens über Emotionalität im Feld als Lehre des Emotions-Managements. ${ }^{51}$ Spätestens mit Renato Rosaldos Thematisierung der Trauer um seine in der gemeinsamen Feldforschungsarbeit verstorbene Frau, Grief and a Headhunter's Rage (1989), und Ruth Behars The Vulnerable Observer (1996) tritt Emotionalität dann an die Oberfläche und kann nicht nur zum Gegenstand ethnologischer Studien, sondern auch zum Thema für eine epistemologische Methodenreflexion werden:52 „Our task is to investigate how certain emotions evoked during fieldwork can be used to inform how we understand the situations, people, communities, and interactions comprising the lifeworlds we enter" 53 schreibt James Davies in der Einleitung des Sammelbandes Emotions in

\footnotetext{
${ }^{49}$ Siehe Malinowski, Bronislaw: A Diary in the Strict Sense of the Term. London 1967; Smith Bowen, Elenore: Return to Laughter. London 1954; Powdermaker, Hortense: Stranger and Friend. The Way of an Anthropologist. New York 1966.

${ }^{50}$ Zur Kritik an ,fieldwork accounts', auch bei James Clifford (,fables of rapport') und Mary Louise Pratt, siehe Reed-Danahay, Deborah: Autobiography, Intimacy, and Ethnography. In: Paul Atkinson u. a. (Hg.): Handbook of Ethnography. London u. a. 2001, S. 407-419, hier S. 412 f.

${ }^{51}$ Siehe Wax, Rosalie H.: Doing Fieldwork. Warnings and Advice. Chicago/London 1975. Vgl. auch Devereux, Georges: Angst und Methode in den Verhaltenswissenschaften. Frankfurt a. M. 1984 (engl. 1967). Devereux sieht affektive Subjektivität allein durch die Subjekt-Objekt-Interaktion in den Verhaltenswissenschaften als immer schon gegeben an: „Die Erkenntnis zwingt uns [...], die Vorstellung aufzugeben, die Grundoperation der Verhaltenswissenschaft sei die Beobachtung eines Objekts durch einen Beobachter. An ihre Stelle muß die Vorstellung treten, daß es um die Analyse der Interaktion zwischen beiden geht, wie sie in einer Situation stattfindet, in der beide zugleich für sich Beobachter und für den anderen Objekt sind“" (S. 309). Subjektivität könne methodisch nie vollständig ,herausgefiltert" werden und erfordere daher in ihren affektiven Aspekten eine ethnopsychoanalytische Untersuchung des Beobachters selbst und seiner interaktionalen Beziehung zum Beobachteten. Devereux' Forderung nach einer ,realistischen Verhaltensforschung“ (S. 21), die auf dem Weg zu einer möglichst objektivierbaren Aussage über ihre Objekte die „Berücksichtigung der emotionalen Verstrickung des Untersuchenden mit seinem Material“ (S. 28) benötige, impliziert mit ihrem Fokus auf Angst jedoch noch immer eine Vorstellung von Emotionalität als psychoanalytisch zu bewältigendes „Hindernis“.

${ }^{52}$ Siehe Rosaldo, Renato: Introduction: Grief and a Headhunter's Rage. In: Ders.: Culture and Truth: The Remaking of Social Analysis. Boston 1989; Behar, Ruth: The Vulnerable Observer. Anthropology That Breaks Your Heart. Boston 1996. Etwa zeitgleich mit diesen Veröffentlichungen plädieren Lila Abu-Lughod und Catherine A. Lutz für eine Diskurs- und soziokulturelle Analyse von Emotionalität, die als ,anthropology of emotions“ ein bis dahin vorherrschendes Verständnis von Emotionen als internen, irrationalen und natürlichen Phänomenen ablösen soll. Abu-Lughod, Lila/Lutz, Catherine A.: Introduction: Emotion, Discourse, and the Politics of Everyday Life. In: Dies. (Hg.): Language and the Politics of Emotion. Cambridge 1990, S. 1-23, hier S. 4.

${ }^{53}$ Davies, James: Introduction: Emotions in the Field. In: James Davies/Dimitrina Spencer (Hg.): Emotions in the Field. The Psychology and Anthropology of Fieldwork Experience. Stanford 2010, S. 1-33, hier S. 1.
} 
the Field und plädiert für eine Rückholung der Emotionalität von den methodologischen Rändern der sozial- und humanwissenschaftlichen Feldforschung. Aus dem ,Reflexive Turn' des Faches müsse zwangsläufig endlich eine Hinwendung zu einem bislang vernachlässigten Thema erfolgen, das in Davies' Darstellung zu einem Potenzial für die ethnologische Forschung werden kann:

Our objective is to show how certain emotions, reactions, and experiences that are consistently evoked in fieldworkers, when treated with the same intellectual vigour as our empirical work demands, can more assist than impede our understanding of the lifeworlds in which we set ourselves down. ${ }^{54}$

Diesem Plädoyer für eine Auseinandersetzung mit der epistemologischen Rolle der Affekte der Forschenden im Feld schließt sich nun auch die vorliegende literaturwissenschaftliche Studie an. Während die Ethnologie jedoch nach einer systematischen Selbst-Reflexion ruft, die methodisch fruchtbar gemacht werden soll, ${ }^{55}$ setze ich den Fokus meiner Arbeit anders: Zwar ist das Anliegen ebenfalls, Emotionalität als „epistemische[n] Schlüssel“56 der Feldforschung zu heben. Statt um die Ethnologie geht es mir jedoch um ihre Schwesterndisziplin, die Primatologie; statt um die praktische und methodische Selbst-Reflexion um die Emotionalität der disziplinär ,Anderen ‘.57 statt um die methodische Nutzbarmachung von Emotionalität im Feld um eine grundlegende Analyse der Thematisierung, Darstellung und Bedingungen von Emotionalität in Narrativen von der Feldforschung und ihre wissenschaftshistorische, poetologische, epistemologische, kulturelle und literarische Kontextualisierung.

Diese Studie geht dabei also nicht nur erstens von der These aus, dass Emotionalität eine epistemologische Rolle in der Forschung im Feld und bei der Generierung primatologischen Wissens spielt. Sie fügt ihr drei weitere Leitthesen bei: Zweitens nimmt sie an, dass emotionale Erfahrungen und Formen der Darstellung der Forschungsarbeit und -ergebnisse in Bezug zueinander stehen. Drittens lassen sich, wie bereits die Diskussion des Paradigmas der wissenschaftlichen Objektivität zeigt, Wahrnehmung, Thematisierung und Bewertung von Emotionalität nur im Kontext übergreifender wissenschaftlicher, kultureller, gesellschaftlicher und auch politischer Bedingungen verstehen, die ,Gefühlskonjunkturen ${ }^{58}$

\footnotetext{
${ }^{54}$ Davies: Introduction, S. 1.

${ }^{55}$ Siehe auch Stodulka, Thomas: Towards an Integrative Anthropology of Emotion. A Case Study from Yogyakarta. In: Anne Storch (Hg.): Consensus and Dissent. Negotiating Emotion in the Public Space. Amsterdam/Philadelphia 2017, S. 9-34; Stodulka, Thomas: Spheres of Passion - Fieldwork, Ethnography, and the Researcher's Emotions. In: Curare 38/1 + 2 (2015), S. 103-116.

${ }^{56}$ Lubrich/Stodulka/Liebal: Affekte im Feld, S. 182.

${ }^{57}$ Vgl. hierzu auch Suter, Fermin/Shah, Mira: Bericht aus dem Feld. Von den Problemen und Vorteilen interdisziplinärer Literaturwissenschaft am Beispiel des Projekts ,Die Affekte der Forscher'. In: Caroline Frank/Daniel Kazmeier/Markus Schleich (Hg.): An den Grenzen der Disziplinen. Literatur und Interdisziplinarität. Hannover 2018, S. 117-137.
}

${ }^{58}$ Landweer/Newmark: Zur Rhetorik von Emotionsdebatten, S. 81 f. 
steuern. Viertens schließlich lässt sich im Austausch von Science und Fiction ein ,Wissen der Literatur ${ }^{59}$ über die Rolle von Emotionalität in der Forschung untersuchen: Spätestens seit Pierre Boulles La planète des singes bedienen sich Literatur und Film nicht nur am jeweils zeitgenössischen Wissen der Primatologie, sondern reflektieren auch die Primatologie mit ihrem kultur- und biologiegeschichtlich besonderen Tier-Mensch-Verhältnis als affektive bzw. affektinduzierende Wissenschaft, indem sie bspw. die emotionalen Konsequenzen zur Aufführung bringen. ${ }^{60}$ Zudem entsteht die Primatologie nicht aus einem wissenschaftlichen Vakuum heraus, sondern schließt an eine Geschichte der Affen(be)forschung an, von der sie ebenso lernt wie sich abzugrenzen bemüht ist.

Diese Leitthesen und ihre Interdependenz strukturieren die Studie: Im ersten Teil (Affektive Epistemologien des Feldes) wird die Frage nach der epistemologischen und ermöglichenden Rolle der Emotionalität im Feld und ihren Dramaturgien in den populärwissenschaftlichen Primärtexten im Vordergrund stehen; im zweiten (Affektpoetik der Forschungsmemoiren) die Formen (Genres und Modi), die das Berichten von Emotionalität und Erfahrungen im Feld in den an ein breiteres Publikum gerichteten Forschungsmemoiren einnimmt; und der dritte Teil der Arbeit (Affektregime der Primatologie) untersucht die wissenschaftlichen, gesellschaftlichen, kulturellen und politischen Bedingungen, vor deren Hintergrund sich die Äußerungsmöglichkeiten von und für Emotionalität bewegen. Diese drei Teile beginnen jeweils mit der Betrachtung eines historischen Vorspiels zu Affen und Affenforschung und enden mit einer Analyse der Funktion von literarischer oder filmischer Reflexion für die Emotionalität der primatologischen Feldforschung.

\subsection{Gattungsfragen}

Warum nun die Primatologie und nicht die Ethnologie, die das Programm eines Studiums der Affekte der Forschenden maßgeblich vorbereitet und angeregt hat? Warum die Konzentration auf die Feldforschung und nicht das Laborexperiment? Warum die Forschungsmemoiren und nicht wissenschaftliche Monographien und Aufsätze oder die Artikel des National Geographic Magazine? Was hat es mit den Affen als affektiv besonderen Tieren auf sich? Und was will die Literaturwissenschaft zur Affektforschung der Science Studies beitragen?

\footnotetext{
${ }^{59}$ Die Studie reiht sich damit in das gleichnamige Forschungsfeld ein, siehe Borgards, Roland/ Neumeyer, Harald/Pethes, Nicolas/Wübben, Yvonne (Hg.): Literatur und Wissen. Ein interdisziplinäres Handbuch. Stuttgart/Weimar 2013; Köppe, Tilmann (Hg.): Literatur und Wissen. Theoretisch-methodische Zugänge. Berlin/New York 2011; Willis, Martin: Literature and Science. A Reader's Guide to Essential Criticism. London/New York 2015.

${ }^{60}$ Ein frühes Beispiel hierfür ist Samuel Becketts Pantomimentext Acte Sans Parole I (1955), bzw. Act Without Words I (1956), der deutlich auf Wolfgang Köhlers Intelligenzprüfungen an Anthropoiden (1917) referiert. Siehe dazu Anm. 47 in Abschn. 4.1.
} 
Im Folgenden soll auf all diese m. E. kaum voneinander $\mathrm{zu}$ trennenden ,Gattungsfragen' eingegangen werden. Erstens wird die Primatologie in ihrer Eigenschaft als Schwesterdisziplin der Ethnologie und lohnenswertes, da noch unterforschtes Untersuchungsfeld für die Beschäftigung mit den Affekten der Forschenden herausgearbeitet. Zweitens soll ein Exkurs zum besonderen Verhältnis des Menschen zum Affen zeigen, inwiefern die Affenforschung der westlichen Welt ${ }^{61}$ in der Tradition eines affektiv ambivalenten Mensch-Tier-Verhältnisses steht. Drittens wird dargelegt, warum eine literatur- und kulturwissenschaftliche Herangehensweise für die Beschäftigung mit einer (populär-)wissenschaftlichen Textform und ihrer Rezeption im Medium von Literatur und Film nicht allein sinnvoll, sondern notwendig ist.

\subsubsection{Im Feld': Primatologie und Feldforschung}

Eine epistemologische, methodische und historische Verwandtschaft von Ethnologie und Primatologie als Teilbereiche einer umfassenden , big tent"-Anthropo$\operatorname{logie}^{62}$ (mit so unterschiedlichen Sektionen wie Sozialanthropologie, Physischer oder Biologischer Anthropologie, Primatologie, Kulturanthropologie, aber auch Archäologie/Paläoanthropologie und Linguistik), die den Menschen zugleich als Kultur- und als evolutionäres Wesen betrachtet, wurde schon verschiedentlich diskutiert. ${ }^{63}$ Im Rahmen der Wissenschaftsforschung in der Primatologie selbst wird z. B. die Suche nach einem ,primate pattern', das Affen und Menschen umfasst und erklärt, sowie die Übernahme von anthropologischen Erklärungs-Modellen kritisch reflektiert. ${ }^{64}$ Donna Haraway sieht in ihrer kulturwissenschaftlichen

\footnotetext{
${ }^{61}$ Diese Studie beschäftigt sich aus Gründen der sprachlichen Zugänglichkeit und kulturellen Expertise mit den Forschungsmemoiren der westlichen Primatologie. Zur japanischen Primatologie siehe aber Asquith, Pamela J.: Anthropomorphism and the Japanese and Western Traditions in Primatology. In: James G. Else/Phyllis C. Lee (Hg.): Primate Ontogeny, Cognition and Social Behavior. Cambridge 1986, S. 61-71; Asquith, Pamela J.: Negotiating Science. Internationalization and Japanese Primatology. In: Shirley C. Strum/Linda Marie Fedigan (Hg.): Primate Encounters: Models of Science, Gender, and Society. Chicago 2000, S. 165-183; Takasaki, Hiroyuki: Traditions of the Kyoto School of Field Primatology in Japan. In: Shirley C. Strum/ Linda Marie Fedigan (Hg.): Primate Encounters: Models of Science, Gender, and Society. Chicago 2000, S. 151-164. Zur indischen Primatologie siehe Haraway, Donna: Primate Visions: Gender, Race, and Nature in the World of Modern Science. New York/London 1989, S. $244 \mathrm{ff.}$

${ }^{62} \mathrm{Vgl}$. MacClancy, Jeremy/Fuentes, Agustín: Centralizing Fieldwork. In: Dies. (Hg.): Centralizing Fieldwork. Critical Perspectives from Primatology, Biological and Social Anthropology. New York/Oxford 2011, S. 1-26, hier S. 2.

${ }^{63}$ Siehe bspw. Strier, Karen B.: Why Anthropology Needs Primatology. In: General Anthropology 18/1 (2011), S. 1-8. Zu einer Kritik ,holistischer" anthropologischer Ansätze siehe aber Segal, Daniel A./Yanagisako Sylvia J. (Hg.): Unwrapping the Sacred Bundle. Reflections on the Discipline of Anthropology. Durham/London 2005.

${ }^{64} \mathrm{Vgl}$. Strum, Shirley C./Fedigan, Linda Marie: Changing Views of Primate Society: A Situated North American View. In: Dies. (Hg.): Primate Encounters. Models of Science, Gender, and Society. Chicago 2000, S. 3-49, hier S. 7 ff.
} 
Auseinandersetzung mit der Primatologie nicht nur die Biologie und Anthropologie im 18. und 19. Jahrhundert als vergleichbaren „locus of highly productive discourses and other social practices" 65 die weiterhin nachwirken, sondern weist unter dem Stichwort des , simian orientalism ‘ auf die Parallelen in den Erkenntniszielen einer vorreflexiven Ethnologie und der modernen Primatologie hin. Wie die frühe Ethnologie versuchte, den westlichen Menschen aus dem oder in Abgrenzung zum ethnologisch ,Anderen“ zu erhellen, verfährt zunächst auch die Primatologie: „Simian orientalism means that western primatology has been about the construction of the self from the raw material of the other, [...] the ripening of the human from the soil of the animal" ${ }^{66}$ Die Primatologie generiere, zumindest in ihrem Fokus auf die evolutionäre Geschichte der Gattung Mensch, in erster Linie ,bioanthropological origin stories “67 für den Menschen, so Haraway.

In den letzten beiden Dekaden hat aber besonders eine zentrale methodische Gemeinsamkeit dazu geführt, dass die reflexiven Anliegen der Ethnologie zu programmatischen Forderungen auch für die Primatologie werden konnten: ${ }^{68}$ Feldforschung ist die ,central method of research throughout anthropology, a much-valued, much-vaunted mode of generating information". ${ }^{69}$ In der Ethnologie durch Bronislaw Malinowski propagiert und von dessen Schüler/innen institutionalisiert, wird die ,participant observation'-Form der Feldforschung in den 1950er Jahren auch für eine gerade entstehende Freiland-Primatologie methodisch vielversprechend. ${ }^{70}$ Es sind vor allem zwei Figuren der Primatologiegeschichte, die diese Forderung nicht nur äußern, sondern auch in eigenen Schulen umsetzen:

\footnotetext{
${ }^{65}$ Haraway: Primate Visions, S. 290.

${ }^{66}$ Haraway: Primate Visions, S. 11. Siehe dazu, wie in europäischen Gesellschaften menschliche Identität traditionell ,in terms of animal alterity“ artikuliert werde, auch Corbey, Raymond: The Metaphysics of Apes: Negotiating the Animal-human Boundary. Cambridge 2005, S. 29.

${ }^{67}$ Haraway: Primate Visions, S. 317.

${ }^{68}$ Vgl. Asquith, Pamela J.: Natural Homes: Primate Fieldwork and the Anthropological Method. In: Jeremy MacClancy/Agustín Fuentes (Hg.): Centralizing Fieldwork. Critical Perspectives from Primatology and Biological Anthropology in the Lens of Social Anthropological Theory and Practice. New York/Oxford 2010, S. 242-255, hier S. 243: „,[A]nthropology is an appropriate home for primatology, based as much on some common fundamentals of methodology as for reasons of evolutionary kinship with humans".

${ }^{69} \mathrm{MacClancy/Fuentes:} \mathrm{Centralizing} \mathrm{Fieldwork,} \mathrm{S.} 1$.

${ }^{70}$ Zum Paradigma malinowskischer Ethnographie siehe Fuchs/Berg: Phänomenologie der Differenz, S. 24 ff. MacClancy/Fuentes weisen darauf hin, dass neben „fieldwork Malinowsky-style“ auch andere, häufig kulturell bedingte Formen der Feldforschung existieren, vgl. MacClancy/ Fuentes: Centralizing Fieldwork, S. 7. Malinowski selbst habe, so Karl-Heinz Kohl, die teilnehmende Beobachtung am Vorbild der Naturwissenschaften und ihres Feld-Begriffs als „eine räumlich begrenzte Einheit, deren voneinander abhängige Einzelelemente stetig aufeinander einwirken und daher nur in ihrem Gesamtzusammenhang studiert werden können“, entwickelt. Kohl: Ethnologie, S. 111.
} 
In den USA propagiert 1951 der ,physical anthropologist“71 Sherwood Washburn eine ,truly biological anthropology [as] comparative studies of free ranging nonhuman primates“. ${ }^{2}$ Der britische Paläoanthropologe Louis Leakey implementiert diese Forderung nach ,long-term (one year or more) coexistence with the population of study“73 als ,hallmark of a majority of primatology dissertations“"74 sogleich in Nähe seiner eigenen Ausgrabungs- und Tätigkeitsstätte in Kenia, indem er die wohl bekannteste Primatologin der Welt ,ins Feld' schickt: Jane Goodall. Leakeys Wahl von Goodall für die Erforschung von Schimpansen, sowie in den Jahren darauf von Dian Fossey für jene der Gorillas und Biruté Galdikas für die der Orang-Utans setzte eine spektakuläre Zäsur für die Erforschung von Affen - und stellt in gewisser Weise frühere und parallel laufende Feldforschungsprojekte in den Schatten. ${ }^{75}$ In der Annahme, dass theoretisch-praktisch ,unprofessionelle' Personen weiblichen Geschlechts geeigneter dazu seien, teilnehmende Beobachtung unter Affen auszuführen, schickt Louis Leakey ab den 1960er Jahren drei Frauen ,ins Feld', die - zugespitzt formuliert - nicht nur die Verhaltensforschung, sondern auch die Sicht des Menschen auf seine nächsten Artverwandten, die anderen Primaten, und auf die Primatenforschung selbst

\footnotetext{
${ }^{71}$ MacClancy/Fuentes: Centralizing Fieldwork, S. 6. Der Mangel an einem eindeutigen deutschen Begriff für diese Unterkategorie der Anthropologie verweist auf die unterschiedliche Entstehungsgeschichte der Primatologie in der US-amerikanischen, britischen, deutschen, französischen oder japanischen Wissenschaftskultur. Vgl. dazu Haraway: Primate Visions, S. 203 ff. MacClancy/Fuentes sehen die US-amerikanische Primatologie personell aus der Anthropologie bestückt, die europäische Primatologie dagegen eher in der Tradition der Tierethologie von Konrad Lorenz und Nikolaas Tinbergen und die japanische Primatologie der Kinji-Imanishi-Schule als ,hybrid of ethnographic, ethological and ecolocial approaches contexutalized in a particular complex and holistic view of nature". MacClancy/Fuentes: Centralizing Fieldwork, S. 8. In der vorliegenden Studie steht die mit Affen im Freiland arbeitende Primatologie im Vordergrund, wie sie in all diesen Wissenschaftskulturen in der einen oder anderen Form praktiziert wird. Davon ist eine experimentell im Labor arbeitende Primatologie abzugrenzen, die teilweise in Kooperation, teilweise jedoch auch in skeptischer Konkurrenz mit der Freiland-Primatologie existiert. Vgl. dazu Lubrich/Stodulka/Liebal: Affekte im Feld, S. 185 ff.

${ }^{72}$ MacClancy/Fuentes: Centralizing Fieldwork, S. 6. Zu Washburn siehe auch Haraway: Primate Visions, S. $203 \mathrm{ff}$.

${ }^{73}$ MacClancy/Fuentes: Centralizing Fieldwork, S. 6.

${ }^{74}$ MacClancy/Fuentes: Centralizing Fieldwork, S. 6 f.

${ }^{75}$ Primatologisch wertvolle Freiland-Studien wurden bereits zuvor von ,nicht-professionellen* Beobachter/innen ausgeführt: Detaillierte Affen-Beobachtungen finden sich in Reiseberichten, Aufzeichnungen von Kolonialbeamten, Hobby-Naturalisten u. ä. Goodalls Feld-Einsatz gehen Feldaufenthalte von George Schaller in Ostafrika sowie vor allem von Clarence Ray Carpenter voraus. Siehe MacClancy/Fuentes: Centralizing Fieldwork, S. 15 ff.; Haraway: Primate Visions, S. 84 ff. Die Washburn-Schule (zu der im Rahmen dieser Arbeit Shirley C. Strum und Sarah Blaffer Hrdy zählen) prägt die US-amerikanische Primatologie über die Universitäten in Harvard und Berkeley, trat jedoch überwiegend weniger medial auf als Leakeys Forscherinnen.
} 
revolutionieren. ${ }^{76}$ Sie tun dies zunächst durch die Etablierung von Forschungsstationen und die Durchführung von Langzeitstudien, die die Feldforschung als Methode in der Primatologie verankern:

\begin{abstract}
The primatologist traditionally goes to live with his or her subjects, pitching camp where the monkeys, apes or lemurs are, watching them day in and day out in an attempt to understand the inner workings and general patterns of the particular primate ,society ${ }^{\text {c }}$ of interest. While not being able to question their informants [...], primatologists seek descriptive answers in the behaviour and inter-individual relationships observable in their primate subjects. ${ }^{77}$
\end{abstract}

Wie MacClancy und Fuentes 2011 in ihrem programmatischen Sammelband zu einer methodischen Reflexion der Feldforschung als dem zentralen Forschungsund Erfahrungsparadigma hier anführen, übernimmt die Primatologie eine in der Ethnologie entwickelte Methode - und damit auch deren Charakteristika, Herausforderungen und epistemischen Besonderheiten: Feldforschung ist zeitintensiv, „,not a state but an open ended process““ ${ }^{78}$ der sich häufig unabhängig von vorformulierten Forschungszielen und wissenschaftlichen Theorien entwickelt und Durchhaltefähigkeit, Kreativität, ethisches Abwägen und Flexibilität erfordert. Besonders aber ist zu berücksichtigen, dass die Methode der ,participant observation'-Feldforschung das wahrnehmende Subjekt ins Zentrum rückt: Die teilnehmende Beobachtung ,has been defined precisely as the use of a person as the research instrument ${ }^{\text {" }}{ }^{79}$ Die persönliche Erfahrungskomponente der Forschung soll systematisch zur Gewinnung objektiver Erkenntnis genutzt werden. ${ }^{80}$ Als solche trägt die Feldforschung - ironischerweise implementiert in einer Episteme der Suche nach der Objektivierung wissenschaftlicher Erkenntnis bei gleichzeitiger Problematisierung des erkennenden Subjektes - in sich immer eine bereits in der Formulierung teilnehmende Beobachtung implizierte Spannung. Goslinga und Frank sehen die Forderung der ,participant observation" als inhärent problematisch für das Subjekt des Wissens, suggeriert sie doch eine unmögliche Spaltung desselben in einen beobachtenden und einen teilnehmenden Teil: „On the one hand, we are called to maintain critical distance and, on the other, to plunge again and

\footnotetext{
${ }^{76}$ Vgl. Morell, Virginia: Called ,Trimates', Three Bold Women Shaped Their Field. In: Science 260/5106 (1993), S. 420-425. Jane Goodalls Bedeutung für die primatologische Feldforschung beschreibt bspw. die Primatologin Allison Jolly als ,lightning rod“ und ,Humanisierung “ der Disziplin durch die Berücksichtigung individueller tierlicher Persönlichkeiten. Siehe Jolly, Allison: The Bad Old Days of Primatology. In: Shirley C. Strum/Linda Marie Fedigan (Hg.): Primate Encounters. Models of Science, Gender, and Society. Chicago 2000, S. 71-84, hier S. 82.

${ }^{77}$ MacClancy/Fuentes: Centralizing Fieldwork, S. 6.

${ }^{78} \mathrm{MacClancy/Fuentes:} \mathrm{Centralizing} \mathrm{Fieldwork,} \mathrm{S.} 10$.

${ }^{79}$ Goslinga, Gillian/Frank Gelya: In the Shadows: Anthropological Encounters with Modernity. In: Athena McLean/Annette Leibing (Hg.): The Shadow Side of Fieldwork. Exploring the Blurred Borders between Ethnography and Life. Malden u. a. 2007, S. xi-xviii, hier S. xii, Hervorheb. MS
}

${ }^{80}$ Vgl. Fuchs/Berg: Phänomenologie der Differenz, S. 31. 
again into the living world“" ${ }^{81}$ Dieser Spaltung korrespondiert ihrer Ansicht nach die Trennung der Kategorien Arbeit und Leben in der Moderne, worin sie mit Michel Foucaults und Bruno Latours Analysen des ,specifically European knot of culture, history and power" ${ }^{* 82}$ konvergieren:

[A]11 forms of embodied and living knowledges are policed in order to uphold the purity of the representation and the sovereignty of the ,subject that knows': emotions, aesthetics, affinities, moral feelings and urges, and, when contrary to national and institutional regimes, political commitments and actions. ${ }^{83}$

In der Feldforschung, gerade jener , unter Affen ‘ in der ,Wildnis ‘ fernab menschlicher Ansiedlungen, fallen aber diese Kategorien - Leben und Arbeit - unmittelbar zusammen und produzieren die Mehrdimensionalität der Feldforschung als Methode, die sie auch für Außenstehende interessant macht, wie sich zeigen wird.

Während die Ethnologie dank ,Reflexive Turn' und Repräsentationskrisen seit geraumer Zeit diskutiert, inwiefern die Forderung nach wissenschaftlicher Objektivität im oben bereits diskutierten Sinn einer Läuterung des Wissenssubjekts von seiner Subjektivität weder haltbar, noch sinnvoll ist, ja autoritäre Verengungen des Wissbaren produzieren kann, wird für die Primatologie eine Diskussion der Feldforschung als Methode und multidimensionaler Prozess jüngst erst angestoßen. Die Forderungen nach einer Auseinandersetzung mit den Bedingungen und Voraussetzungen, unter denen primatologisches Wissen im Feld produziert wird, antworten auf Kritik aus den vor allem experimentell arbeitenden Bereichen der Primato$\operatorname{logie^{84}}$ und konzentrieren sich zunächst noch auf die kulturellen, methodologischen und wissenschaftshistorischen Aspekte der Feldforschung. ${ }^{85}$ Obwohl in diesen Auseinandersetzungen mit der zentralen Methode der primatologischen Freilandforschung auch emotionale Aspekte eine Rolle spielen, bleibt die affektive Dimension, gerade in Verbindung mit der Rolle des Tieres nicht nur als Forschungsobjekt sondern auch als Interaktionspartner/in für die Feldforschenden noch unterbelichtet. Dies überrascht angesichts der kulturhistorisch bereits gut erforschten affektiven Rolle des Affen zumindest in der westlichen (anthropologischen) Imagination, vor deren Hintergrund sich die moderne Affenforschung bewegt.

\footnotetext{
${ }^{81}$ Goslinga/Frank: In the Shadows, S. xv.

${ }^{82}$ Goslinga/Frank: In the Shadows, S. xvi; Foucault, Michel: Die Ordnung der Dinge: Eine Archäologie der Humanwissenschaften. Frankfurt a. M. 1974 (frz. 1966); Latour, Bruno: Wir sind nie modern gewesen. Versuch einer symmetrischen Anthropologie. Frankfurt a. M. 42013 (frz. 1991).

${ }^{83}$ Goslinga/Frank: In the Shadows, S. xvi.

${ }^{84}$ Vgl. Lubrich/Stodulka/Liebal: Affekte im Feld, S. 185 f.; Fedigan, Linda Marie: Primate Paradigms. Sex Roles and Social Bonds. Chicago/London 1992, S. 17.

${ }^{85}$ Siehe neben MacClancy/Fuentes bspw. Forderungen nach einer Ethik der Feldforschung und einer Ethnoprimatologie: Malone, Nicolas M./Fuentes, Augustín/White, Francis J.: Ethics Commentary: Subjects of Knowledge and Control in Field Primatology. In: American Journal of Primatology 72 (2010), S. 779-784; Fuentes, Agustín/Hockings, Kimberley J.: The Ethnoprimatological Approach in Primatology. In: American Journal of Primatology 72/10 (2010), S. 841-847.
} 


\subsubsection{Exkurs: Von Affen und Menschen}

In Flauberts Erzählung Quidquid Volueris (1810/1837) tritt im 8. Kapitel in einigen Ausführungen zur Vorliebe älterer Damen für Schoßhunde und Hausaffen überraschend ein ,Ich` wieder zutage, welches seit Beginn der Erzählung von einer auktorialen Perspektive verdrängt schien. Dieses Ich offenbart:

Je n'aime guère des singes, et pourtant j'ai tort, car ils me semblent une imitation parfaite de la nature humaine. Quand je vois un de ces animaux (je ne parle pas ici des hommes), il me semble me voir dans les miroirs grossissants: mêmes sentiments, mêmes appétits brutaux, un peu moins d'orgueil et voilà tout. ${ }^{86}$

Flauberts Erzählung über ein Halbwesen aus Mensch und Affe dringt in die Empfindungswelt einer hybriden Kreatur inmitten der bürgerlichen Pariser Gesellschaft ein. Was an dieser Stelle überrascht, ist nicht allein die bekenntnishafte Aussage. Bezeichnend ist auch der Einschub ,je ne parle pas ici des hommes“, als sei es an dieser Stelle vonnöten, die Unterscheidung von Affen und Menschen zu betonen - um doch im nächsten Satz genau zwischen diesen eine irritierende Ähnlichkeit herzustellen. Die Struktur dieser Ähnlichkeit ist die der satirischen Imitation. Im Affen erkennt sich der Mensch unangenehm wieder - „et voilà tout.“ Ganz so einfach ist das Verhältnis des Menschen zu seinen nächsten Artverwandten natürlich nicht. Aber Flaubert bringt in seiner Erzählung in dieser Form zum Ausdruck: Dieses Mensch-Tier-Verhältnis ist ein besonderes, und es ist geprägt von starken Überzeugungen, Faszination und Ängsten, in denen das Ich in seinen Affekten zutage tritt.

Mit der Bedeutung des Affen für den Menschen und der Besonderheit dieses Tier-Mensch-Verhältnisses hat sich die Forschung bereits ergiebig befasst. Roland Borgards' und Julika Griems literaturwissenschaftliche Studien beschäftigen sich zum Beispiel mit der Rolle von Affen als Ordnungstieren und literarischen Tropen. ${ }^{87}$ Hans Werner Ingensieps philosophische Kulturgeschichte des Affen

\footnotetext{
${ }^{86}$ Flaubert, Gustave: Quidquid volueris. In: Ders.: Euvres de jeunesse. Hg. von Claudine Gothot-Mersch/Guy Sagnes. Paris 2001 (Euvres complètes, Bd. 1), S. 241-272, hier S. 265.

${ }^{87}$ Siehe Borgards, Roland: Affen. Von Aristoteles bis Soemmerring. In: Roland Borgards/Günter Oesterle/Christine Holm (Hg.): Monster: zur ästhetischen Verfassung eines Grenzbewohners. Würzburg 2009, S. 239-252; Borgards, Roland: Der Affe als Mensch und der Europäer als Ureinwohner. Ethnozoographie um 1800 (Cornelis de Pauw, Wilhelm Hauff, Friedrich Tiedemann). In: David E. Wellbery (Hg.): Kultur-Schreiben als romantisches Projekt. Romantische Ethnographie im Spannungsfeld zwischen Imagination und Wissenschaft. Würzburg 2012, S. 17-42; Borgards, Roland: Hund, Affe, Mensch. Theriotopien bei David Lynch, Paulus Potter und Johann Gottfried Schnabel. In: Maximilian Bergengruen/Roland Borgards (Hg.): Bann der Gewalt: Studien zur Literatur- und Wissensgeschichte. Göttingen 2009, S. 105-142; Borgards, Roland: Menschenaffen/Affenmenschen. In: Michael Gamper/Martina Wernli/Jörg Zimmer (Hg.): „Es ist nun einmal zum Versuch gekommen“. Experiment und Literatur I: 1580-1790. Göttingen 2009, S. 293-308; Griem, Julika: Monkey Business: Affen als Figuren anthropologischer und ästhetischer Reflexion 1800-2000. Berlin 2010.
} 
vollzieht nach, wie Wissen über Affen generiert und kulturell kontextualisiert wird. ${ }^{88}$ Horst-Jürgen Gerigk, Virginia Richter und Donna Haraway haben sich mit einzelnen Zeiträumen und der Nutzbarmachungen des Affen als kulturelles, literarisches und auch biologisches Tier befasst. ${ }^{89}$ Der Kulturanthropologe Raymond Corbey schließlich arbeitete eine Metaphysik der Affen heraus, welche im anthropologischen Diskurs die Sammlung und Interpretation von Daten beeinflusst. ${ }^{90}$ An dieser Stelle soll daher mit Verweis auf diesen Forschungsstand auf einen genauen historischen Abriss zur Kenntnis und Bedeutung der Affen weitgehend verzichtet werden. Stattdessen möchte ich anhand einer Verortung des Affen als Figur menschlicher Selbstreflexion in der westlichen Welt herausarbeiten, weshalb das Verhältnis des Menschen zu den Affen als seinen Mitprimaten ein emotional aufgeladenes ist.

Die Ähnlichkeit zwischen menschlichen und nicht-menschlichen Primaten benötigt nicht erst eine systematische anatomische, physiologische oder neurologische und ethologische Forschung am Affen, um sich als signifikant für anthropozentrische Betrachtungen zu erweisen. Seit der Antike sind Affen belegt mit der „Hybris der Nachahmung““ ${ }^{91}$ Besonders im Mittelalter und der frühen Neuzeit lässt diese sündhafte Imitationsfähigkeit Affen als Figuren in einer ästhetischen Auseinandersetzung darum dienen, wie sich ,echte', da originäre, und ,falsche ', da nur nachahmende Kunst, unterscheide. Diese Tradition, in der im Mittelalter auch der Teufel als simia Dei, als Affe/Nachahmer Gottes betrachtet wird, ${ }^{92}$ lässt sich noch im englischen Adjektiv , simian“ für , affenartig ' nachvollziehen, spielt für die vorliegende Studie jedoch eine untergeordnete Rolle. ${ }^{93}$ Sie besitzt zwar auch

\footnotetext{
${ }^{88}$ Siehe Ingensiep, Hans Werner: Der kultivierte Affe: Philosophie, Geschichte und Gegenwart. Stuttgart 2013; Ingensiep, Hans Werner: Der aufgeklärte Affe. Zur Wahrnehmung des Menschenaffen im 18. Jahrhundert im Spannungsfeld zwischen Natur und Kultur. In: Jörn Garber/Heinz Thoma (Hg.): Zwischen Empirisierung und Konstruktionsleistung: Anthropologie im 18. Jahrhundert. Berlin/Boston 2004, S. 31-58.

${ }^{89}$ Siehe Gerigk, Horst-Jürgen: Der Mensch als Affe in der deutschen, französischen, russischen, englischen und amerikanischen Literatur des 19. und 20. Jahrhunderts. Hürtgenwald 1989; Richter, Virginia: Literature after Darwin: Human Beasts in Western Fiction, 1859-1939. Basingstoke 2011; Haraway, Donna: Monkeys, Aliens, and Women: Love, Science, and Politics at the Intersection of Feminist Theory and Colonial Discourse. In: Women's Studies International Forum 12/3 (1989), S. 295-312; Haraway: Primate Visions.

${ }^{90}$ Corbey: The Metaphysics of Apes.

${ }^{91}$ Krüger, Gesine/Mayer, Ruth/Sommer, Marianne: Figuren des Dazwischen. Menschenaffen und Affenmenschen als Grenzwesen. Eine Einleitung. In: Dies. (Hg.): „Ich Tarzan. “Affenmenschen und Menschenaffen zwischen Science und Fiction. Bielefeld 2008, S. 7-20, hier S. 9.

${ }^{92}$ Vgl. Borgards: Affen, S. 240.

${ }^{93}$ Siehe hierzu aber Janson, Horst Woldemar: Apes and Ape Lore: In the Middle Ages and the Renaissance. London 1952; Sirc, Susan: Monkeys, Monuments and Miracles: Aspects of Imitation of Word and Image in Die Wahlverwandtschaften. In: German Life and Letters 47, S. 432-448; Boa, Elizabeth: Aping and parroting: Imitative performance in Goethe's Die Wahlverwandtschaften. In: Carolin Duttlinger/Lucia Ruprecht/Andrew Webber (Hg.): Performance and Performativity in German Cultural Studies. Oxford u. a. 2003, S. 21-40.
} 
emotionalisierende Komponenten, besonders in der christlich motivierten Ablehnung des Affen als Sündentier und in den ästhetischen Debatten des 18. Jahrhunderts. Sie verweist den Affen jedoch durch die Abstraktion seiner Fähigkeiten in die Kunst hinein und schreibt ihm als moralischer Allegorie weitaus weniger emotionale Ambivalenz zu, als dies durch seine umstrittene wesenhafte Stellung in einer anthropozentrisch hierarchisierten Ordnung geschieht. Auch bei dieser Neubesetzung des Affen in der Neuzeit spielen die Fertigkeiten und Befähigungen der unterschiedlichen Affenarten eine große Rolle; diese Charakteristika werden jedoch zunehmend aufs Ganze eines definitiven Unterscheidungskriteriums hin gedacht. ${ }^{94}$

Bereits Aristoteles sinnierte über die Menschenähnlichkeit der Affen, ${ }^{95}$ doch erst im 17. Jahrhundert wird der Affe zum Gegenstand einer naturwissenschaftlich-philosophischen Auseinandersetzung mit der Frage, was der Mensch sei. Zentral für diese und die sich anschließende Frage, wie der Mensch sich von anderen Wesen systematisch unterscheiden lasse, sind, wie der Titel Edward Tysons markiert, Menschenaffen: Orang-Outan, sive Homo Sylvestris; Or, The Anatomy of a Pygmie compared with That of a Monkey, an Ape, and a Man (1699). ${ }^{96}$ Denn von einer kolonialen Welt-Erweiterung, entdeckt' und den philosophierenden Anatomen ab dem 17. Jahrhundert auf die Seziertische und in die Orangerien fürstlicher Höfe getragen, ist die epistemologische Einordnung dieser sogenannten Menschenaffen zwischen zoologischen und mythologischen Ordnungssystemen lange ungewiss. ${ }^{97}$ So werden sie zu Medien menschlicher Verunsicherung: Carl von Linnés Zusammenführung der Affen und Menschen als erst ,Anthropomorpha“ (1735), dann ,Primates“ (1758) in der taxonomischen Ordnung seines Systema Naturae ${ }^{98}$ bringt die erste, Darwins primatische Erbschaftsfolge in der Evolutionstheorie seines On the Origin of Species $^{99}$ (1859) die zweite große Erschütterung in einem Bewusstsein, das den Menschen zunächst in einer Kette, dann in einer Stufenleiter, schließlich in einem Baum, aber immer teleologisch an der Spitze verortet. Virginia Richter spricht im Zusammenhang dieser zweiten Erschütterung von einer fundamentalen Kategoriekrise, die sich in anthropologischer Angst (,anthropological anxiety') entlade. Diese Verunsicherung betrifft für Richter ,a cultural definition of ,the human' based on an attempt to separate the anthropos from the non-human“. ${ }^{100}$ Menschenaffen, die den Menschen durch

\footnotetext{
${ }^{94}$ Vgl. Wild: Anthropologische Differenz.

${ }^{95}$ Siehe Aristoteles: Historia Animalium. Berlin 2013, S. 45 f.

${ }^{96}$ Tyson, Edward: Orang-outang, sive, Homo sylvestris: or, the Anatomy of a Pygmie Compared with that of a Monkey, an Ape, and a Man [1699 Facs.]. London 1966.

${ }^{97}$ Vgl. Borgards: Affen, S. 244 ff.; sowie Krüger/Mayer/Sommer: Figuren des Dazwischen; und auch Borgards: Menschenaffen/Affenmenschen.

${ }^{98}$ von Linné, Carl: Systema naturae. Regnum animale. Stockholm ${ }^{10} 1758$.

${ }^{99}$ Darwin, Charles: On the Origin of Species by Means of Natural Selection: or the Preservation of Favoured Races in the Struggle of Life. London 1859.

${ }^{100}$ Richter: Literature after Darwin, S. 8, Hervorheb. im Original.
} 
Form und Befähigung synchron mit der Vielfalt an anderen Primatenformen ${ }^{101}$ und diachron mit den hominiden Vorformen ${ }^{102}$ verbinden, machen diese Ordnungen und Erklärungsmodelle aber möglich, indem sie sich als epistemologische, bewegliche Figuren nutzen lassen: Mehr noch als andere Affen sind sie Bewohner eines Grenzgebietes zwischen den mythischen Polen Kultur und Natur. ${ }^{103}$ Sie sind als Phantasmen einer rassen- und speziesübergreifenden Sexualität und als poetologische und biographische Reflexionsfiguren sowie als Figuren der Internalisierung und Regression ,liminale Kreaturen“, ${ }^{104}$ wie Julika Griem beschreibt. Für Roland Borgards wird der von ihnen bewohnte Grenz- oder Schwellenraum erst durch die Differenzierung von Mensch und Tier produziert:

\begin{abstract}
Wie ein kleines logisches Maschinchen produziert die Differenzierung zwischen Mensch und Tier einen Dritten Raum, eine limitrophe Zone, der [sic] weder dem Animalischen noch dem Humanen ohne Schwierigkeiten zugeordnet werden kann. Es gibt Menschen; es gibt Tiere; und es gibt ein Drittes. Dieses Dritte stellt als unmögliches aber dennoch wirkliches tertium datur ein fundamentales epistemologisches Problem dar. Von dieser Beobachtung aus lässt sich die theriotopische Position des Affen, die Position des Affen in einer kulturellen Tier-Raum-Ordnung beschreiben: Der Affe ist ein Grenzbewohner. ${ }^{105}$
\end{abstract}

Wie Borgards' Ausführungen zur theriotopischen Position des Affen zeigen, lässt sich der Affe, und hier speziell der Menschenaffe, ausgiebig nutzen als philosophische Allegorie und theoretische Figur. ${ }^{106}$ Bei Borgards wird diese Einordnung des Affen mithilfe von Konzepten der Postcolonial Studies (der Dritte Raum) und der Dekonstruktion (die limitrophe Zone) vorgenommen. Julika Griem verwendet zusätzlich die Begriffe der Identität und Alterität, um die Nutzbarmachung des Affen als Figur zu verdeutlichen: Für Griem dienen, sie demonstriert dies anhand von überwiegend literarischen Texten, Affen als kulturelle Figurationen von Identität und Alterität. Damit belegen die Studien dieser Literaturwissenschaftler/innen über die semantische Verwertung, kulturelle Kontextualisierung und philosophische Allegorisierbarkeit von Affen selbst Raymond Corbeys für die philosophische Anthropologie aufgestellte These, Affen seien ,good to think and symbolize with“, 107 und Donna Haraways Beobachtung zur Meta-Funktionalität der Affen: „Primates existing at the boundaries of so many hopes and interests are wonderful subjects with whom to explore the permeability of walls, the reconstruction of boundaries, the distaste for endless socially enforced dualisms." 108

\footnotetext{
${ }^{101}$ Vgl. Richter: Literature after Darwin, S. 14.

${ }^{102}$ Vgl. Krüger/Mayer/Sommer: Figuren des Dazwischen, S. 10; Corbey: The Metaphysics of Apes, S. 60 ff.

${ }^{103}$ Vgl. Haraway: Primate Visions, S. 1.

${ }^{104} \mathrm{Vgl}$. Griem: Monkey Business, S. 88.

${ }^{105}$ Borgards: Affen, S. 242, Hervorheb. im Original.

${ }^{106}$ Ingensiep sieht dies als Grundvoraussetzung für das menschliche Interesse am Affen. Vgl. Ingensiep: Der kultivierte Affe, S. $15 \mathrm{ff}$.

${ }^{107}$ Corbey: The Metaphysics of Apes, S. 6.

${ }^{108}$ Haraway: Primate Visions, S. 3.
} 
Ob Grenz- und Schwellenbewohner, ,Missing Link' in der Stufenleiter aller Wesen, ${ }^{109}$ Alteritäts- und Identitätsfiguren, Affen bedeuten meist mehr als sie sind, indem sie immer wieder für etwas anderes als sich selbst herangezogen werden. Dabei sind sie jedoch keinesfalls nur ,rationale“ Denkfiguren, sondern gerade wegen ihrer relativen und nicht absoluten Alterität ${ }^{110}$ - aufgrund ihrer prekären Stellung als, um hier Homi Bhabhas Formulierung zu kapern, ,almost the same, but not quite" 111 menschlich - mit emotionaler Ambivalenz aufgeladen. Aus dem Affen ströme die ,theriotopische Unruhe des gegebenen Dritten“, schreibt Borgards: „Er ist kein Tier wie die anderen, sondern ein menschenähnliches, weshalb die Abgrenzung besonders nötig und mit besonderer rhetorischer Energie betrieben wird“. ${ }^{112}$ Dies zeigt sich besonders deutlich in einem anthropologischen Diskurs, der seinem Wesen als Beschäftigung mit dem Menschen nach auch immer ein anthropozentrisch organisierter ist. Durch die Nähe des Affen zum Menschen ist er immer wieder ein Faktor der Bedrohung von identitätsstiftenden Ordnungen, zugleich aber ein Artefakt des ebenfalls Identität konstruierenden Wissens über den Menschen. In diesem Sinn sind Affen sogar zentrale Akteure anthropologischer und kulturphilosophischer Diskurse und erlangen durch die ihnen eignende Ambiguität eine eigene Wirkungsmacht als „,powerful carriers of meaning and emotion". ${ }^{113}$ Diese zeigt sich vor allem in ihrer Wirkung auf das emotionale Befinden des Menschen, je nachdem, welchen Nutzen Affen als philosophische, anthropologische, literarische oder zoologisch-ökologische Tiere erfüllen. Die von Borgards, Richter und Corbey besprochene ontologische Unruhe, tief verwurzelt in der Verunsicherung, die von ihrer Menschenähnlichkeit, und der Bedrohung, die von ihrer Physis ausgeht, ist dabei eine intellektuell induzierte Seite des emotionalen Befindens, das sich in panischer Distinktionsangst ebenso äußern kann wie in vagem Unwohlsein, aufgeweckter Neugier oder wohlwollender Empathie. Dabei wandelt sich das Ansehen der Affen durch verschiedene wissenschaftliche und gesellschaftliche Paradigmen hinweg mehrfach, z. B. allein im von Julika Griem untersuchten Zeitraum von 1800 bis 2000 von der abgewehrten Bestie zum anerkannten und geschätzten , alter ego '. ${ }^{114}$ Für diesen Wandel wesentlich verantwortlich ist die Primatologie als Tochterwissenschaft der Anthropologie: In dem Maß, in dem Affen auch für sich eigenständig und ,ökologisch valide" beobachtet und zur Erschließung der menschlichen Evolution herangezogen sowie als durch die Zerstörung ihres Lebensraums und Wilderei limitierte und bedrohte Ressource wahrgenommen werden, werden sie zu

\footnotetext{
${ }^{109}$ Vgl. Krüger/Mayer/Sommer: Figuren des Dazwischen, S. 9 ff.

${ }^{110} \mathrm{Vgl}$. Borgards: Affen, S. 252.

${ }^{111}$ Bhabha, Homi: The Location of Culture. London/New York 2004, S. 123, Hervorheb. im Original.

${ }^{112}$ Borgards: Affen, S. 252.

${ }^{113} \mathrm{Vgl}$. Corbey: The Metaphysics of Apes, S. 25.

${ }^{114}$ Vgl. Griem: Monkey Business, S. 25.
} 
emotional und evolutionär Verbündeten: „Nowhere in the study of the animal world, it would seem, is the emotional involvement of the human species so great as with its own ancestry and next of kin“, ${ }^{115}$ schreibt Raymond Corbey zu Beginn seiner Studie. Er weist jedoch auch darauf hin, inwiefern diese jüngsten Entwicklungen - fort vom ,Othering' und hin zum ,Selfing' des Affen - ebenfalls Teil einer stetigen Pendelbewegung zwischen Bestialisierung und Humanisierung in der Betrachtung des Affen aus anthropologisch-anthropozentrischer Perspektive heraus ist. ${ }^{116}$ Die emotional fundierten Überzeugungen zum Affen sieht Corbey dabei geprägt von der Aufgabe, die die Figur Affe im jeweiligen epistemischen wissenschaftlichen und gesellschaftlichen Paradigma einnimmt: Bedrohung der menschlichen Ausnahmestellung im Tierreich oder Erkenntnismedium über die Entwicklung des Menschen; Erinnerung an das animalische Wesen der menschlichen Natur oder idealisierte Vorstellung eines von der Zivilisation unverdorbenen Naturzustands; Verkörperung des aus dem Ich in die koloniale Peripherie ausgelagerten ,Other' oder empathisch angeeignetes und durch rhetorische Angleichung konstruiertes prähistorisches ,Wir“ im Tier?

Es lässt sich somit auf Basis der Forschungsliteratur zur Rolle des Affen für den Menschen festhalten: 1) Affen nehmen in den Weiten einer menschlichen Imagination vom Tier und seiner Bedeutung einen besonderen Raum ein, der sie von anderen epistemischen Nutztieren unterscheidet und ihnen eine zentrale Rolle im Mensch-Tier-Verhältnis verleiht. 2) Die Basis dafür bildet die Menschenähnlichkeit, die ihnen anatomisch, genetisch, behavioral und sozial eignet - oder qua anthropomorphisierendem ,Default-Setting ' des menschlichen Denkens zugeeignet wird. ${ }^{117} 3$ ) Affen werden nicht nur als Artefakte wissenschaftlicher Unterscheidung und als Versuchstiere für Erkenntnis über den Menschen, sondern auch als Denkfiguren, besonders als philosophische und kulturelle Allegorien genutzt. 4) Sie lassen sich in dieser Funktion nicht auf eine diskursive Bestimmung oder wertende Verortung festlegen, denn sie sind als Denkfiguren mobil und ambig. 5) Gerade diese Ambiguität in Verbindung mit den Distinktionsbemühungen und -ängsten anthropologischer Identitätsfindung verleiht ihnen eine starke emotionale Komponente. 6) Die ambivalente Emotionalität dieses - so ist anzunehmen - unilateralen Mensch-Affe-Verhältnisses prägt wissenschaftliche und philosophische Debatten und Diskurse, aber auch literarische Auseinandersetzungen mit dem Affen.

\footnotetext{
${ }^{115}$ Corbey: The Metaphysics of Apes, S. 6.

${ }^{116} \mathrm{Vgl}$. Corbey: The Metaphysics of Apes, S. 1.

${ }^{117}$ Zum Anthropomorphismus als epistemischer Grundeinstellung menschlichen Denkens siehe Daston, Lorraine/Mitman, Gregg (Hg.): Thinking with Animals: New Perspectives on Anthropomorphism. New York 2004; Wild, Markus: Anthropomorphismus. In: Arianna Ferrari/Klaus Petrus (Hg.): Lexikon der Mensch-Tier-Beziehungen. Bielefeld 2015, S. 26-28. Zur Politik des Anthropomorphismus-Vorwurfs siehe Abschn. 4.1 und 4.3 der vorliegenden Studie.
} 


\title{
1.3.3 Science/Fiction
}

Die primatologische Feldforschung erhöht dieses ambivalente Emotionalisierungspotenzial der Affen: Erstens, indem sie neue Erkenntnisse über die ,nächsten Artverwandten` zutage fördert, die, wie bereits Marianne Sommer festgestellt hat, das Potenzial besitzen, das menschliche Selbstverständnis grundlegend zu erschüttern:

\begin{abstract}
These fields define what it means to be human, what we regard as our evolutionary heritage, and which kinds of behaviour and social structures (of domination and subordination) we consider to be natural and thus resistant to change. The disciplines of physical anthropology, primate ethology and social ecology have an impact on our concept of self (and other) and thus seem most prone to generate ideologically motivated knowledge. Where our origin is at stake, expectations, fears and projections must be at their maximum. ${ }^{118}$
\end{abstract}

Die als Prozess der Wissensgenerierung tradierte Verschriftlichung der primatologischen Feldforschung narrativiert und dramatisiert zweitens die Prozesse des Forschens und der Interaktion mit den Tieren, einzelne tierliche Lebensgeschichten und persönliche menschliche Erfahrungen und verwendet damit potenziell emotionsauslösende rhetorische bzw. literarische Verfahren in der Darstellung von Wissen.

Die Primatologie sucht schon früh ein Publikum jenseits der akademischen Welt und weiß dieses mit Repräsentationen der Feldforschung für das Fach und seine tierlichen Akteure/innen zu interessieren. ${ }^{119}$ Es sind gerade die von Louis Leakey eingesetzten Forscherinnen, die durch die mediale Vermittlung ihrer Beobachtungen und Erkenntnisse und durch ihre Texte - Dissertationen, Fachaufsätze, bunt bebilderte Artikel und Dokumentarfilme für die National Geographic Society, aber vor allem autobiographische, populärwissenschaftliche Forschungsberichte (Forschungsmemoiren) - einer breiteren Öffentlichkeit nicht nur die Feldforschung als ,exciting adventure of self-realization and academic advance“120 darstellen. Sie geben vor allem auch emotionales Zeugnis von den Erfahrungen der Feldforschung. Diese ,narrative Primatologie ' geht für die Anthropologin und Primatologie-Theoretikerin Pamela Asquith ,to the heart of the fieldwork experience for primatologists studying animals in their natural habitats“. ${ }^{121}$ Forschungsmemoiren inszenieren affektive Begegnungen und

\footnotetext{
${ }^{118}$ Sommer, Marianne: Foremost in Creation. Anthropomorphism and Anthropocentrism in National Geographic Articles on Non-Human-Primates. Bern u. a. 2000, S. 10, Hervorheb. MS. ${ }^{119} \mathrm{Vgl}$. MacClancy/Fuentes: Centralizing Fieldwork, S. $21 \mathrm{ff}$.

${ }^{120}$ MacClancy/Fuentes: Centralizing Fieldwork, S. 21. Zum spezifischen Nexus der Goodall-Fossey-Galdikas-Primatologie im Feld und ihrer Medialisierung siehe Noble: Politics, Gender, and Worldly Primatology; Morell: Called ,Trimates'.

${ }^{121}$ Asquith: Natural Homes, S. 243.
} 
skizzieren bedeutungsvolle Beziehungen zwischen den menschlichen Wissenschaftler/innen und den tierlichen Beforschten; sie arbeiten gestalterisch tierliche Lebensgeschichten heraus und binden diese in (auto)biographische Dramaturgien ein, die ihnen im Rahmen des Forschungsprozesses Sinn verleihen.

Weil diese Texte im Gegensatz zu wissenschaftlichen Aufsätzen in Fachzeitschriften oder Dissertationsmonographien, die an einzelnen Forschungsinteressen ausgerichtet, durch methodische und theoretische Ansätze bestimmt oder im Umfang stark limitiert sind, aus dem Vollen der Forschungserfahrung schöpfen können, bieten sie, so ist der Forschung bereits aufgefallen, analog zu den ,ethnographic memoirs'122 (bzw. ,confessional tales', ,fieldwork accounts' oder ,personal narratives $\left.{ }^{6}\right)^{123}$ der Ethnologie eine breitere und tiefere Einsicht in das, was Feldforschung als, reale‘ Erfahrung ist:

The narrative accounts, however, are exceptional in that they provide sustained reports on the fieldwork experience and hence very much richer examples about methodology, about the nature of the relationship between the observers and animals, and that among the animals themselves. These books are not confined to describing the ,ethnographic process" in fieldwork: they are part personal memoir, part research report, and part enriched descriptive accounts of whole animal lives. ${ }^{124}$

Auch MacClancy und Fuentes teilen Asquiths Augenmerk für die narrativen Berichte von der Feldforschung in der Primatologie und fordern: „,[]f we wish to understand the development of primatology in a rounded manner then we need to examine how literary-minded primatologists have expressed themselves to others. "125

Hier setzt die vorliegende Studie an: Im Zentrum stehen Forschungsmemoiren als eben jene ,narrative accounts of primatology', die nicht allein von einzelnen Feldforschungsaufenthalten berichten, sondern einen Bezug zur Forschendenbiographie

\footnotetext{
${ }^{122}$ Siehe Tedlock, Barbara: From Participant Observation to the Observation of Participation: The Emergence of Narrative Ethnography. In: Journal of Anthropological Research 47/1 (1991), S. 69-94.

${ }^{123}$ Vgl. zur Vielfalt von Begriffen für Formen, die sich an der Peripherie des ,traditionellen Kanons wissenschaftlichen Schreibens bewegen Reed-Danahay: Autobiography, Intimacy, and Ethnography; Okely, Judith: Anthropology and autobiography. Participatory experience and embodied knowledge. In: Judith Okely/Helen Callaway (Hg.): Anthropology \& Autobiography. London/New York 1992, S. 1-28, hier S. 10 ff.; Pratt, Mary Louise: Fieldwork in Common Places. In: James Clifford/George E. Marcus (Hg.): Writing Culture. The Poetics and Politics of Ethnography. Berkeley/Los Angeles 1986, S. 27-50; Golde: Introduction; Leibing, Annette/ McLean, Athena: ,Learn to Value Your Shadow!' An Introduction to the Margins of Fieldwork. In: Dies. (Hg.): The Shadow Side of Fieldwork. Exploring the Blurred Borders between Ethnography and Life. Malden u. a. 2007, S. 1-28, hier S. 7 ff.

${ }^{124}$ Asquith: Natural Homes, S. 243.

${ }^{125}$ MacClancy/Fuentes: Centralizing Fieldwork, S. 22, Hervorheb. MS.
} 
herstellten. ${ }^{126}$ Geht es der Reflexion innerhalb der Anthropologie darum, an diesen Texten Aufschluss über Methodologie und Erfahrungsqualitäten der Feldforschung $\mathrm{zu}$ gewinnen, ist es Anliegen der vorliegenden Studie, als literatur- und kulturwissenschaftliches Projekt danach $\mathrm{zu}$ fragen, in welchem Zusammenhang Feldforschung und Emotionalität im Medium von Text stehen.

Dabei sollen nicht nur erstmals die primatologischen Forschungsmemoiren als eigenständiges Genre untersucht werden. ${ }^{127}$ Am Beispiel der Primatologie kann dabei auch ins Auge gefasst werden, dass und wie eine empirisch basierte Wissenschaft Erkenntnis nicht nur aus der Interpretation und Anordnung von ,Daten“ produziert, sondern auch Repräsentation, Narration, Dramatisierung und ähnlich ,literarische“ Verfahren verwendet, um sowohl Wissen $\mathrm{zu}$ formen, $\mathrm{zu}$ normieren und $\mathrm{zu}$ veranschaulichen, als auch - besonders im Fall der Forschungsmemoiren - Erfahrungen und Eindrücke aus der Forschungsarbeit zu vermitteln. Analog zur ethnologischen Idee des ,Writing Culture' lässt sich in Bezug auf die Primatologie von ,Writing Science' sprechen: ${ }^{128}$ Die Forschungsmemoiren sind nicht nur eine literarisierende Ausdrucks-, sondern auch eine Produktionsweise von Wissen.

Für die Primatologie kann damit seitens der Literaturwissenschaft eine Reflexionsarbeit angeboten werden, wie sie der ethnologische Strang der Anthropologie im Rekurs auf Literatur- und Kulturtheorien des 20. Jahrhunderts

\footnotetext{
${ }^{126}$ Diese narrativen Berichte sind insofern Memoiren, als sie erzählerisch eine nicht-fiktionale Repräsentation der Erfahrungen eines realen Menschen bieten, ohne den Anspruch zu erheben, ein menschliches Leben in Gänze in den Mittelpunkt zu stellen. Vielmehr bieten sie Raum für Gedanken, Erinnerungen und Beobachtungen, die u. a. eine bestimmte Zeit einer individuellen Biographie betreffen. Vgl. Couser, G. Thomas: Memoir. An Introduction. Oxford u. a. 2012, S. 7 ff.; Wagner-Egelhaaf, Martina: Autobiographie. Stuttgart 2005, S. 6; Lejeune, Philippe: Der autobiograhische Pakt. Frankfurt a. M. 1994 (frz. 1975), S. 14.

${ }^{127}$ Mary Sanders Pollock macht zwar ebenfalls , field narratives‘ der Primatologie zum Gegenstand ihrer Studie, betrachtet die Primärtexte aber nicht in Hinblick auf ihren Charakter als Forschungsmemoiren, sondern rückt sie in die Nähe eines bachtinschen Roman-Begriffs, um narratologisch vorzugehen. Sie schreibt anhand der ,field narratives' dabei eher eine Entwicklungsgeschichte der Primatologie und ihrer Erzählungen, die die Primatolog/innen und ihre Tiere bzw. Tierarten ins Zentrum setzt und den appellativen Charakter der Artenschutzrhetorik aufnimmt. Vgl. Sanders Pollock, Mary: Storytelling Apes. Primatology Narratives Past and Future. University Park 2015, S. 8 ff.

${ }^{128}$ Unter dem Titel ,Writing Science“ veröffentlichten die Linguisten Michael Halliday und James Martin 1993 eine lexikogrammatische Studie zur Sprache wissenschaftlicher Texte, in der sie ,[t]he evolution of science“ als ,the evolution of scientific grammar" betrachten und somit wissenschaftliches Schreiben von anderen Texten unterscheiden. Dass diese offizielle wissenschaftliche Schreibweise als Stimme der Objektivität rhetorischer Effekt ist und naturwissenschaftliche wie andere Textgenres betrachtet und hinsichtlich ihrer rhetorischen, konstruktiv-produktiven und diskursiven Dimension daraufhin untersucht werden können, wie ,scientific knowledge" hergestellt wird, diskutieren bereits jeweils Locke und Myers. Halliday, Michael A. K./Martin, James R.: Writing Science. Literacy and Discursive Power. London/Washington, D.C. 1993, S. 12; Locke, David: Science as Writing, New Haven/London 1992, S. 112; Myers, Greg: Writing Biology. Texts in the Social Construction of Scientific Knowledge. Madison/London 1990, S. ix.
} 
bereits vollzogen hat, und wie sie aus unterschiedlichen Gründen innerhalb der zunehmend an den ,hard sciences ${ }^{6}{ }^{129}$ sich ausrichtenden Primatologie noch weitgehend aussteht. ${ }^{130}$ Auch wenn dies für Vertreter/innen jener Zweige der experimentell arbeitenden Laborprimatologie eine Zumutung und eine Bestätigung ihrer Vorurteile der Feldprimatologie gegenüber darstellen mag, ${ }^{131}$ schlage ich vor diesem Hintergrund auch für die primatologischen Forschungsmemoiren und ihre Rezeption mit Donna Haraway Science Fiction mit starker Betonung der ,Science“ als Orientierungsbegriff vor. ${ }^{132}$

Überwiegend betrifft der Science-Fiction-Begriff die Interdependenz von ,Technoscience“ und Fiktion. ${ }^{133}$ Weil aber wissenschaftliche Forschung Narrative benötigt, um ihre Ergebnisse zu beschreiben und zu verbreiten, schlägt Manuela Rossini in der Folge Haraways vor, literarische, visuelle, theoretische und populärwissenschaftliche Texte als ,Science/Fiction` zu bezeichnen. Der Schrägstrich ist dabei nicht als Grenzlinie zwischen ,Science“ und ,Fiction“, sondern als Signal für die liminale Existenz solcher Narrative zwischen Wissenschaft und Literatur zu verstehen. Sowohl wissenschaftliche Texte, die narrativ gestaltet sind, als auch Literatur, die wissenschaftliche Inhalte und Errungenschaften aufgreift, sind dabei als unterschiedliche Typen von ,Science/Fiction“ zu betrachten, die zusammen eine kulturelle Matrix formen. Für die technowissenschaftliche ,Science/Fiction", welche Rossini im Kontext posthumanistischer Überlegungen betrachtet, sind solche Texte Grundlage der materiell-semiotischen Konstruktion (Rossini nennt dies in Anlehnung an das Werk der unterschiedlichen Spezialist/innen, die Disneyland

\footnotetext{
${ }^{129}$ Zur Hierarchisierung der Wissenschaften (,hard ' vs. , soft ${ }^{`}$ ) siehe Smith, Laurence D. u. a.: Scientific Graphs and the Hierarchy of the Sciences: A Latourian Survey of Inscription Practices. In: Social Studies of Science 30/1 (2000), S. 73-94.

${ }^{130} \mathrm{Nach}$ der u. a. durch die in der Anthropologie wirksam werdenden Gender und Postcolonial Studies und die Science Studies inspirierten Auseinandersetzung mit den Traditionen und Paradigmen des Feldes Primatologie durch Primatolog/innen selbst (bspw. Fedigan und Strum), scheint seit knapp nach der letzten Jahrhundertwende die reflexive Beschäftigung dank verschiedener theoretischer, struktureller und methodischer Entwicklungen in der Primatologie überwiegend den Geistes- und Sozialwissenschaften überlassen. Siehe Fedigan: Primate Paradigms; Strum, Shirley C./Fedigan, Linda Marie: Theory, Method, Gender, and Culture. What Changed Our Views of Primate Society? In: Shirley C. Strum/Donald G. Lindburg/David A. Hamburg (Hg.): The New Physical Anthropology. Upper Saddle River, N.J. 1999, S. 67-105; Strum, Shirley C./Fedigan, Linda Marie (Hg.): Primate Encounters. Models of Science, Gender, and Society, Chicago 2000. Siehe aber Julia Keil: Wissenschaft und Gefühl. Eine Interviewstudie zum Umgang mit Empfindungen in der Forschung an nichtmenschlichen Primaten. Berlin 2019. https://refubium.fu-berlin.de/handle/fub188/25348 (11.10.2019).

${ }^{131}$ Vgl. Lubrich/Stodulka/Liebal: Affekte im Feld, S. 185 f.

${ }^{132} \mathrm{Vgl}$. Haraway: Primate Visions, S. 5.: ,the narratives of scientific fact - those potent fictions of science - within a complex field indicated by the signifier SF“"

${ }^{133} \mathrm{Vgl}$. Rossini, Manuela: Figurations of Posthumanity in Contemporary Science/Fiction. All Too Human(ist)? In: Revista Canaria de Estudios Ingleses 50 (2005), S. 21-37. Zur Problematik von Science-Fiction-Definitionen siehe auch Freedman, Carl: Critical Theory and Science Fiction. Middletown 2000, S. $14 \mathrm{ff}$.
} 
schufen, ,imagineering ') von Zukünften. In der Primatologie betrifft ,Science/Fiction' im Kontext der Konservation von Habitaten auch das Imaginieren katastrophaler Zukünfte, vor allem aber das ,imagineering' von Vergangenheiten. Roland Borgards hat für solche Narrative evolutionärer menschlicher Vergangenheiten den Begriff Primatographien ${ }^{134}$ geprägt: primatologisches Erzählen von der biologischen Vorgeschichte des Menschen, jene bioanthropologischen Ursprungserzählungen, wie sie u. a. Haraway kritisiert. ${ }^{135}$ Diese unterstehen dem von Albrecht Koschorke in seiner allgemeinen Erzähltheorie für ,kulturell[e] und epistemisch[e] Anfangserzählungen“136 aufgestellten schöpferischen Prinzip:

Was gilt, hängt auf elementare Weise davon ab, welcher Erzählanfang gesetzt wird, der die Gegenwärtigkeit des Erzählten von einer aus dem Innern der narrativen Raumzeit unartikuliert scheinenden, ungeordneten Prähistorie trennt und damit immer auch schon den Gegenstand definiert, der den Kern der Geschichte bildet. ${ }^{137}$

Die primatologischen Texte, die in dieser Studie im Fokus stehen, beinhalten teilweise primatographische Passagen, stellen überwiegend aber das Arsenal an Argumenten und die Datenbasis dar, aus denen die Primatographie entstehen kann. Dennoch gilt für ihre Erzählweisen das, was Borgards für die Primatographie ausführt:

Die Erzählung kann zunächst als supplementäres epistemologisches Verfahren bewertet werden: Die Geschichte illustriert lediglich das Argument; sie plausibilisiert das Wissen, indem sie es schöner, anschaulicher macht, nicht, indem sie es besser macht. Die Erzählung kann sodann als alternatives epistemologisches Verfahren bewertet werden: Geschichte und Argument addieren ihre je eigene Kraft; die Geschichte plausibilisiert das Wissen, indem sie ihm etwas Gleichwertiges hinzufügt (oder auch: entgegenstellt). Und schließlich kann die Erzählung als konkurrierendes epistemologisches Verfahren bewertet werden: Die Geschichte konstituiert das Argument; sie ist das plausibilisierende Fundament des Wissens, auf dem sich Argumente nur zum Schein errichten. ${ }^{138}$

Damit umreißt Borgards prägnant drei fundamentale Wirkungsmechanismen bzw. Verknüpfungstechniken von Geschichte (oder Narrativ) und Argument in primatologischen Schriften, auf die bereits Donna Haraway in Primate Visions hingewiesen hat. ${ }^{139}$ Die meisten Primatolog/innen würden vermutlich - ähnlich

\footnotetext{
${ }^{134}$ Borgards, Roland: Primatographien. Wie Michael Tomasello und Frans de Waal die biologische Vorgeschichte des Menschen erzählen. In: Johannes F. Lehmann/Roland Borgards/ Maximilian Bergengruen (Hg.): Die biologische Vorgeschichte des Menschen: Zu einem Schnittpunkt von Erzählordnung und Wissensformation. Freiburg i. Br. 2012, S. 361-376.

${ }^{135}$ Vgl. Haraway: Primate Visions, S. 315; aber auch Latour, Bruno/Strum Shirley C.: Human social origins: Oh please, tell us another story. In: Journal of Social and Biological Structures 9 (1986), S. 169-187.

${ }^{136}$ Koschorke, Albrecht: Wahrheit und Erfindung: Grundzüge einer Allgemeinen Erzähltheorie. Frankfurt a. M. 2012, S. 396.

${ }^{137}$ Koschorke: Wahrheit und Erfindung, S. 62.

${ }^{138}$ Borgards: Primatographien, S. 362, Hervorheb. im Original.

${ }^{139}$ Vgl. Haraway: Primate Visions, S. 5.
} 
wie die technowissenschaftlichen Forscher/innen, auf die Manuela Rossini eingeht - die Überlegung, sie verwendeten oder benötigten Narrative, von sich weisen. Wissenschaftshistorisch ist es jedoch ein Leichtes nachzuweisen, dass in der Primatologie wissenschaftliche, kulturelle und gesellschaftspolitische Paradigmen starke Narrative ausbilden, die die jeweiligen Argumente und sogar bereits die ihnen zugrunde liegenden Beobachtungen steuern. ${ }^{140}$ Donna Haraway hat der Primatologie als ,a story of correct vision of primate social form“"141 aus diesem Grund eine ganze Studie gewidmet:

Scientific practice may be considered a kind of story-telling practice - a rule-governed, constrained, historically changing craft of narrating the history of nature. Scientific practice and scientific theories produce and are embedded in particular kinds of stories. Any scientific statement about the world depends intimately upon language, upon metaphors. [...] Scientific practice is above all a story-telling practice in the sense of historically specific practices of interpretation and testimony. ${ }^{142}$

Erzählen ist in jedem (also auch im wissenschaftlichen) Fall nicht bloß, so fasst es Albrecht Koschorke, ,eine reproduktive, den erzählten Inhalten gegenüber nachrangige Tätigkeit [...], kein bloßes Rekapitulieren after the fact ",${ }^{143}$ sondern es lässt sich von einer Art epistemologischen Rückkopplung sprechen, in manchen Fällen (besonders der Sozialontologie, an der die Primatologie ihren Anteil hat) ${ }^{144}$ sogar von einer ,performative[n] Rückkopplung zwischen Zeichen und bezeichneten Gegebenheiten“. ${ }^{145}$ Wie von wissenschaftlichen Beobachtungen erzählt wird, ist entscheidend für die Erkenntnisse, die aus der Beobachtung zu gewinnen sind: ,[J]eder epistemologischen Klärung geht eine ästhetische Entscheidung voraus", ${ }^{146}$ wie Joseph Vogl die Basis für ,Poetologien des Wissens“ fasst.

Dabei können wissenschaftliche Paradigmen, gepaart mit den sie umgebenden Vorstellungen von Standards für wissenschaftliche Objektivität und gestützt durch die Sprache und die Metaphern bestimmter Theoriebildung, teilweise ein enges Korsett bilden, das das erzählbare Material rigide einschnürt. In der Beschäftigung

\footnotetext{
${ }^{140}$ Siehe Fedigan: Primate Paradigms; Strum/Fedigan: Changing Views of Primate Society; Strum/Fedigan: Theory, Method, Gender, and Culture. What Changed Our Views of Primate Society?; Latour/Strum: Human social origins.

${ }^{141}$ Haraway: Primate Visions, S. 4.

${ }^{142}$ Haraway: Primate Visions, S. 4, Hervorheb. MS.

${ }^{143}$ Koschorke: Wahrheit und Erfindung, S. 22, Hervorheb. im Original.

${ }^{144}$ Siehe zuletzt bspw. Tomasello, Michael: A Natural History of Human Morality. Cambridge, Mass./London 2016.

${ }^{145}$ Koschorke: Wahrheit und Erfindung, S. 23, Hervorheb. im Original.

${ }^{146}$ Vogl, Joseph: Einleitung. In: Ders. (Hg.): Poetologien des Wissens um 1800. München 1999, S. 7-16, hier S. 13 f.
} 
mit den primatologischen Texten wird sich zeigen, dass Primatolog/innen aus diesem Grund häufig bewusst andere Formate als den akademischen Bericht suchen, um Emotionalität Raum zu gewähren, die in der Forschung und im Verhältnis zum Tier eine prägnante Rolle spielt. Die Forschungsmemoiren bieten einen solchen Raum für die Thematisierung emotionaler Erfahrungen an. Ihre Literarizität transportiert zudem zusätzliche Schichten der Information über Forschungsprozesse und -erkenntnisse, aber auch die Subjekte der Erkenntnis. Zugleich sind die Forschungsmemoiren als Texte, die sich zuallererst an eine breitere Öffentlichkeit richten, Instrumente, um diese Öffentlichkeit für die eigene Wissenschaft und deren Objekte, die Affen, zu interessieren. Emotionalität ist dabei im Sinne rhetorischer und narrativ-dramaturgischer Verfahren nicht nur als Mittel der Unterhaltung, sondern auch zur Überzeugung und Aktivierung des Publikums zum Handeln wirksam.

Wissenschaftliches Erzählen entsteht jedoch auch im Austausch mit nicht-wissenschaftlichen Formen des Erzählens. Im Folgenden sollen deswegen nicht nur die ,Poetologien des Wissens', sondern auch das ,Wissen der Literatur ernst genommen werden. Meiner Ansicht nach fördert das hohe Emotions- und Emotionalisierungspotenzial der Primatologie ihre Aufnahme und Bespiegelung in einem kulturellen und populärkulturellen Bereich. Dessen kreative Räume erlauben z. B. Gedankenexperimente, die in den primatologischen Schriften wegen der Objektivitätspflicht der Wissenschaftlichkeit aus-, oder aber theoretisierend blutleer bleiben. ${ }^{147}$ Literatur produziert in diesem Sinn epistemologische Fiktionen: Texte, die an Wissensdiskursen partizipieren, indem sie einen literarischen Raum für die Reflexion und Darstellung, aber auch die Infragestellung von Wissen und Wissensproduktion bieten. ${ }^{148}$ Dies zeigt sich bereits an Pierre Boulles außerordentlich erfolgreichem Planet-der-Affen-Stoff, ${ }^{149}$ dem aber noch zahlreiche filmische und literarische Texte folgen, die sich mit der Erfahrungsrealität, den Protagonist/ innen, den Erkenntnissen und auch den ethischen Konsequenzen primatologischer Forschung beschäftigen und teilweise Leerstellen füllen, die die primatologischen Texte hinterlassen haben. Aus diesen Erzeugnissen wiederum lernen Primatolog/

\footnotetext{
${ }^{147}$ Zur Verwandtschaft literarischer und wissenschaftlicher Erkenntnis im Gedankenexperiment siehe Weigel, Sigrid: Das Gedankenexperiment: Nagelprobe auf die facultas fingendi in Wissenschaft und Literatur. In: Thomas Macho/Annette Wunschel (Hg.): Science \& Fiction. Über Gedankenexperimente in Wissenschaft, Philosophie und Literatur. Frankfurt a. M. 2004, S. 183205.

${ }^{148}$ Vgl. Klinkert, Thomas: Epistemologische Fiktionen. Zur Interferenz von Literatur und Wissenschaft seit der Aufklärung. Berlin/New York 2010, S. 5. Nach Klinkert erzeugt Literatur ,in der Regel kein wissenschaftlich valides, neues Wissen von der Welt“, dafür aber ,in der Auseinandersetzung mit vorhandenem Wissen und wissenschaftlichen Prinzipien ein Metawissen“ und ermöglicht „eine Bewusstmachung der Grundlagen des Wissens. In diesem Sinne ist die Literatur keine Wissenschaft oder Philosophie, sondern ein Medium der Erkenntnis von Erkenntnis“ (S. 21).

${ }^{149}$ Siehe auch Shah, Mira: Nach dem Anthropozän: Der Planet der Affen. In: Gabriele Dürbeck/ Jonas Nesselhauf (Hg.): Repräsentationsweisen des Anthropozän in Literatur und Medien/Representations of the Anthropocene in Literature and Media. Berlin u. a. 2019, S. 219-235.
} 
innen, wie sich von der eigenen Forschung und ihren Anliegen erzählen lässt, wie die Forschung eingängig und publikumswirksam wird. ${ }^{150}$

\subsubsection{Wie Primatologie lesen?}

Die Primatologie ist verschiedentlich bereits zum Gegenstand wissenschaftshistorischer, kultur- und literaturwissenschaftlicher Untersuchungen geworden. Am bekanntesten darunter dürfte Donna Haraways hier bereits mehrfach angeführte Studie Primate Visions sein, die untersucht, welche Rolle die Primatologie in der Herstellung einer kulturellen Matrix spielt, die Geschlecht, Rasse und Natur in ein scheinbar natürliches ontologisches Geflecht einspinnt. Gemeinsam mit der Primatologin Shirley C. Strum hat auch der Wissenschaftssoziologe und -philosoph Bruno Latour primatologisches Wissen als Teil der großen wissenschaftlichen Ursprungserzählungen und Primatologie als Gegenstand der Science Studies untersucht. ${ }^{151}$ Auch die drei ,Gründungsfiguren“ der Feldprimatologie, Jane Goodall, Dian Fossey und Biruté Galdikas, waren der Fokus von Veröffentlichungen. ${ }^{152}$ Besondere Faszination übt dabei häufig aufgrund ihres frühen, gewaltsamen Todes und ihrer Forschungspersönlichkeit Dian Fossey aus. ${ }^{153}$

\footnotetext{
${ }^{150}$ Eindrücklich zeigte sich dies an der Kooperation der beiden Primatolog/innen Christophe und Hedwige Boesch mit Disneynature für den Film Chimpanzee im Jahr 2012. Der Film über die Adoption eines verwaisten Jungtieres durch einen männlichen Schimpansen im Taï National Forest in der Elfenbeinküste ist einen Zwitter aus Naturdokumentation und Dokufiktion, für den die Primatolog/innen als Expert/innen zur Verfügung standen, mit dem sie jedoch auch die eigene Forschung zu Altruismus unter Schimpansen an ihrem Feldforschungsort bekannt machen konnten. Siehe Fothergill, Alastair/Linfield, Mark: Chimpanzee. Walt Disney Studios Motion Picture 2012. Zu der in einer solchen Kooperation liegenden Gefahr der ,Disneyfizierung der Primatologie vgl. Mitman, Gregg: Life in the Field: The Sensuous Body as Popular Naturalist's Guide. In: Shirley C. Strum/Linda Marie Fedigan (Hg.): Primate Encounters. Models of Science, Gender, and Society. Chicago 2000, S. 421-435, hier S. 422 ff.

${ }^{151}$ Latour/Strum: Human social origins; Latour, Bruno: A Well-Articulated Primatology: Reflections of a Fellow Traveler. In: Shirley C. Strum/Linda Marie Fedigan (Hg.): Primate Encounters: Models of Science, Gender and Society. Chicago/London 2000, S. 358-381.

${ }^{152}$ Siehe Montgomery, Sy: Walking with the Great Apes. Jane Goodall, Dian Fossey, Biruté Galdikas. Boston 1991; Noble: Politics, Gender, and Worldly Primatology; Bishop, Rebecca: Writing the Body Wild: Primatological Narrative and Spaces of Animality. In: Culture, Theory \& Critique 49/2 (2008), S. 133-148; Griem: Monkey Business; Mitman: Life in the Field; Sowards, Stacy K.: Gender Representations in Orangutan Primatological Narratives. Essentialist Interpretations of Sexuality, Motherhood, and Women. In: Douglas A. Vakoch (Hg.): Ecofeminism and Rhetoric: Critical Perspectives on Sex, Technology, and Discourse. New York 2011, S. 65-88.

${ }^{153}$ Siehe bspw. Mowat, Farley: Woman in the Mists. The Story of Dian Fossey and The Mountain Gorillas of Africa. New York 1987; Norwood, Vera: Made From This Earth. American Women and Nature. Chapel Hill/London 1993, S. 209 ff.; Torgovnick, Marianna: Primitive Passions. Men, Women, and the Quest for Ecstasy. Chicago/London 1998, S. 88 ff.; Whitlock, Gillian: Remediating Gorilla Girl. Rape Warfare and the Limits of Humanitarian Storytelling. In: Biography 33/3 (2010), S. 471-497.
} 
Auch die populärwissenschaftlichen Medien der Primatologie haben bereits wissenschaftliche Aufmerksamkeit gefunden. So widmete sich Marianne Sommer in einer sprachwissenschaftlich ausgerichteten Studie anhand der National-Geographic-Artikel von Primatolog/innen der Rolle von Anthropomorphismen, Anthropozentrismen und Ideologien bei der Generierung von Wissen über nicht-menschliche Primaten. ${ }^{154}$ Filme sind als Medium der Primatologie ebenfalls untersucht worden. ${ }^{155}$ Mit den Qualitäten des ,storytelling ' in den Genres einer ,narrative ethology ' bzw. ,narrative primatology ' haben sich am Beispiel Jane Goodalls aus der Perspektive der Cultural und Literary Animal Studies und mit Fokus auf das Erzählen von Tieren Susan McHugh und aus jener der Literaturwissenschaft mit Fokus auf die spezifische Vermittlungsfunktion für wissenschaftliche, ökologische und konservatorische Anliegen Mary Sanders Pollock beschäftigt. ${ }^{156}$ Zudem wurden, etwa bei Julika Griem, die (populär-)wissenschaftlichen Texte der Primatologie als Interdiskurse zur Analyse von literarischen Texten genutzt. ${ }^{157}$ Obwohl das Affektive dabei immer wieder eine (auch problematische) Rolle gespielt hat, ${ }^{158}$ ist eine Untersuchung des Genres der Feldforschungsmemoiren der Primatologie mit dem Fokus auf Emotionalität aus epistemologischer, poetologischer und kulturanalytischer Perspektive jedoch noch nicht erfolgt.

Die vorliegende Studie bietet nun also erstens der Primatologie eine ähnliche literatur- und kulturtheoretisch informierte Reflexion ihrer Produktions- und Vermittlungsbedingungen, wie diese in der Ethnologie bereits erfolgt ist und z. T. zu einer Wende zum Affektiven geführt hat. Zweitens hebt sie dabei mit den Forschungsmemoiren ein Genre, das sich paradoxerweise sowohl an den Rändern der Wissenschaftlichkeit zu bewegen als auch essenziell für die Wissensproduktion im Feld, die Selbstrepräsentation von Forschenden und die Popularität der Primatologie als Disziplin zu sein scheint. Die Studie untersucht nicht nur

\footnotetext{
${ }^{154}$ Siehe Sommer: Foremost in Creation.

${ }^{155}$ Siehe Hediger, Vinzenz: Was will Jane im Dschungel? Film als Medium der Primatologie von Tarzan bis Jane Goodall. In: Gesine Krüger/Ruth Mayer/Marianne Sommer (Hg.): „Ich Tarzan. “ Affenmenschen und Menschenaffen zwischen Science und Fiction. Bielefeld 2008, S. 51-72.

${ }^{156}$ Siehe McHugh, Susan: Animal Stories. Narrating across Species Lines. Minneapolis/London 2011, S. 211 ff.; Sanders Pollock: Storytelling Apes.

${ }^{157}$ Siehe Griem: Monkey Business; Richter, Virginia: Ape Meets Primatologist. Post-Darwinian Interspecies Romances. In: Tina Gianquitto/Lydia Fisher (Hg.): America's Darwin. Darwinian Theory and U.S. Literary Culture. Athens/London 2014, S. 360-387.

${ }^{158}$ Haraway schreibt 1989 bereits zum so komplexen wie ambigen Verhältnis von Emotionalität und Geschlecht in der Primatologie: ,Western scientific women in particular must negotiate their relation to these taboos. On the one hand, many have deliberately taken advantage of the greater latitude for women in western culture to acknowledge emotional exchange with the animals and to affirm the importance of identification or empathy in a way that they believe improves the research. On the other hand, these same women, as well as many who allow no greater identification than their male peers, repeatedly report having to guard against incautious admission or cultivation of their feelings, in order to be respected scientifically or to avoid being labeled ,naturally“ intuitive“. Haraway: Primate Visions, S. 249.
} 
drittens die Erzählung von der Feldforschung und die Ausdrucks- und Produktionsbedingungen von Wissen über Affen und Menschen sowie den darin wirkenden emotionalen Anteil. Sie berücksichtigt in ihrer Funktion als literaturund kulturwissenschaftliche Arbeit auch viertens die kulturhistorische Einbettung der westlichen Primatologie und ihrer Texte in eine lange Auseinandersetzung mit den Affen als dem Menschen besonderen Tieren und fragt nach den Interferenzen von Literatur und Wissen(schaft) bei der Erschließung der Emotionen in der ,Science/Fiction“ primatologischer Forschungsmemoiren und den epistemologischen Fiktionen, in denen die Primatologie tätig wird. Um der Frage nach der komplexen Rolle von Affekten in der Forschung mit Affen im Feld gerecht zu werden, muss das Material in dieser Vierer-Konstellation untersucht werden: Was berichten die Forschungsmemoiren über Emotionalität im Feld und ihre Rolle in epistemologischen Prozessen? Wie werden emotionale Erfahrungen im Text in eigene Darstellungsformen gebracht und wozu dienen diese? Warum ist ein Sprechen/Schreiben über Emotionalität möglich oder nicht möglich? Welche Wechselwirkung besteht zwischen ,Science" und ,Fiction“ über die Forschungsmemoiren hinaus mit anderen Texten, welche Erkenntnis produziert die Fiktion hinsichtlich der primatologischen Emotionalität?

Wie bereits angekündigt strukturiert diese Fragen-Konstellation auch dieses Buch: Drei Haupt-Analyseteile widmen sich jeweils einem Untersuchungsbereich (Epistemologie, Poetologie, Ideologie ${ }^{159}$ ), diskutieren neben ihrem Fokus auf die Forschungsmemoiren jeweils ein historisches ,Vorspiel' der Affenforschung und untersuchen in einem jeweils abschließenden Kapitel die Funktion von Fiktion für primatologische Emotionalität.

Dabei wird sich im ersten Analyseteil der Arbeit hinsichtlich der Affektiven Epistemologien des Feldes zeigen, dass die ,Trimates' weitgehend keine ,neue weibliche Wissenschaft" erfinden, sondern angesichts der aufgrund ihres Geschlechtes an sie gerichteten Erwartungen hinsichtlich ihrer Leistungen in der

\footnotetext{
${ }^{159}$ Ideologie dient hier als Hilfsbegriff, unter den sich Affektregime, Gefühls- und Wissenschaftskulturen sowie kulturelle Diskurse subsumieren lassen, die Wahrnehmungen, Erfahrungen, Denk- und Erkenntnisweisen, Äußerungsmöglichkeiten, Theoriegebäude und Rezeption steuern und prägen. Dabei soll Ideologie also weniger als ,falsches Bewusstsein "verstanden werden, vor allem, weil die Ideologiekritik hier selbst eine emotionsfeindliche Pejorisierung von Gefühl vornimmt: „Jede Ideologie beruht auf der Theoretisierung und Objektivierung eines primären Gefühlsverhältnisses, das zwischen dem Sprechenden und einem Objekt besteht“; „während echte Theorie rein gegenstandsorientiert, objektiv ist, besagt der Ideologievorwurf, daß der Denkende etwas von seiner Subjektivität in die Aussage geschmuggelt hat. Sein Gefühlsverhältnis zu einem Objekt geht als ein angeblich-Objektives in die Aussage ein“, siehe Geiger, Theodor: Ideologie und Werurteile. In: Kurt Lenk (Hg.): Ideologie. Ideologiekritik und Wissenssoziologie. Frankfurt a. M./New York 1984, S. 184-189, hier S: 186 f. Eher darf Ideologie wissenssoziologisch in der Folge Karl Mannheims als unumgängliches Wesensmerkmal der menschlichen Denkstruktur, als jeweils historisch und kulturell spezifische ,geistige Lebensluft [...], in welcher das erkennende Subjekt atmet", verstanden werden. Lenk, Kurt: Problemgeschichtliche Einleitung. In: Ders. (Hg.): Ideologie. Ideologiekritik und Wissenssoziologie. Frankfurt a. M./New York: Campus 1984, S. 13-49, hier S. 42.
} 
Affenbeobachtung im Feld auf eine traditionelle westliche affektive Epistemologie und Rhetorik rekurrieren und diese produktiv machen können. Damit berücksichtigt die Arbeit eine Tendenz des Diskurses um die ,Trimates ' kritisch, die mit Rekurs auf das biologische Geschlecht der Forschenden die Charakteristika ihrer Forschungsarbeit und ihrer Persönlichkeitsstruktur im Repertoire kulturell kodifizierter Weiblichkeitsstereotype (,das Feminine') verankert. Diese Tendenz verdankt sich nicht allein Louis Leakeys ideologischen Auswahlprinzipien für seine Forschenden, sondern auch den Anliegen einer historischen feministischen Aufwertung weiblicher (Emotions-)Arbeit. Bei Sy Montgomery beginnt bspw. der Forschungsansatz von Goodall und in Folge von Fossey und Galdikas „by relinquishing control“. ${ }^{160}$ Dieser Ansatz sei das Andere einer ,masculine world of Western science, where achievement is typically measured by mastery“. ${ }^{161}$ Montgomery geht daher soweit, einen solchen Ansatz als ,particularly feminine“ zu bezeichnen, denn: „This approach allows choice and the nurturing of a relationship on the Other's term". ${ }^{162}$ Hier liegt m. E. eine problematische affirmative Übernahme der Termini soziokulturell kodierter und zugespitzter Geschlechterideale, maskulin und feminin, vor. Montgomery mag versuchen, aus der Not eine Tugend zu machen - dabei verpasst sie aber die Chance, eben dieses Verhältnis von Wissenschaft und Geschlechterideal näher zu beleuchten. Evelyn Fox Keller hat bereits darauf hingewiesen, inwiefern in der Frühen Neuzeit mit Francis Bacons Revolutionierung des wissenschaftlichen Denkens die Natur als weiblich konzipiert und deren Erkundung paradigmatisch durch ihre Beherrschung ersetzt wurde. Keller legt dar, wie mit der Normierung einer wissenschaftlichen Praxis im Dienste der Entwicklung eines Ideals objektiver Wissenschaft vor allem in der Natur- aber in zunehmendem Maße auch in den Humanwissenschaften die Ausbildung eines Männlichkeitsideals einhergeht. Dem setzt Fox Keller mit einer Nahanalyse der Arbeitsweisen und Vorstellungen der Genetikerin Barbara McClintock die Alternative einer Forschungsweise entgegen, die jedoch eher in Teilen ,das Andere der Vernunft ' berücksichtigt, als sich als spezifisch weibliche Wissenschaft zu verstehen - geschweige denn sich einem als Anderes der Maskulinität gedachten Ideal wie der Femininität zu verschreiben. ${ }^{163}$ Die von Montgomery benannten Charakteristika eines solchen Ideals, Kontrollabtretung (,relinquishing of control“) und Beziehungspflege (,nurturing of a relationship“), entsprechen verdächtigerweise denn auch den klassischen ,weiblichen“ Tugenden besonders in der Ehe, Bacons Lieblingsmetapher für die Beziehung zwischen Forschendem und Natur. Eine Aufwertung von Emotionalität unter derart ,ideologischen' Kriterien läuft daher häufig Gefahr, paradoxerweise eine Lesart zu unterstützen, die

\footnotetext{
${ }^{160}$ Montgomery: Walking with the Great Apes, S. xvi.

${ }^{161}$ Montgomery: Walking with the Great Apes, S. xvi.

${ }^{162}$ Montgomery: Walking with the Great Apes, S. xvi, Hervorheb. MS.

${ }^{163}$ Vgl. Fox Keller: Reflections, S. 33 ff. u. 158 ff; Böhme, Hartmut/Böhme, Gernot: Das Andere der Vernunft. Zur Entwicklung von Rationalitätsstrukturen am Beispiel Kants. Frankfurt a. M. 1983. Zu einer Kritik Montgomerys siehe auch Torgovnick: Primitive Passions, S. 108.
} 
Emotionalität als Sentimentalität abwertet und die das epistemologische Interesse der Emotionsforschung sowie die Verdienste der betreffenden Forscherinnen und der ,Methode Feldforschung ' für die Primatologie zu unterlaufen droht.

Als Vorbereitung für die Beschäftigung mit den Affekten im Feld soll deswegen zunächst einleitend zu diesem Analyseteil ein Blick auf ältere Modelle der affektiven Affenbegegnung geworfen werden, wie sie sich im Forschungs- und Abenteuerbericht der Reiseliteratur finden lassen (Abschn. 2.1). Anhand zweier in diesem Punkt einflussreicher Texte sollen dabei zwei Modelle voneinander abgegrenzt werden, die als Pole eines affektiven Spektrums betrachtet werden können. Der Afrikareisebericht Explorations and Adventures (1861) des Abenteurers Paul Du Chaillu steht dabei für das Modell der sentimentalen Jagd; der Forschungs- und Reisebericht The Malay Archipelago (1869) des neben Charles Darwin zeitgenössisch wichtigsten Evolutionstheoretikers und Naturforschers Alfred Russel Wallace für das Modell einer weitgehend affektlosen, nüchternen Sammeltätigkeit. Im Anschluss wird es in Abschn. 2.2 darum gehen, wie die zeitgenössisch mit dem Spitznamen ,Trimates` belegten Gründungsfiguren der Feldprimatologie, Jane Goodall, Dian Fossey und Biruté Galdikas, vor dem Hintergrund dieser älteren Modelle für die Emotionalität der Reise und der Tierbegegnung sich das Vorurteil zur Kopplung von Emotionalität und Geschlecht zu eigen machen, um für die Forschung im Feld eine affektbasierte Methodik und Epistemologie zu entwickeln. Anhand der Ausformung einer von Donna Haraway als ,Drama of Touch ${ }^{164}$ bezeichneten Dramaturgie des taktilen Erstkontaktes lässt sich diskutieren, wie die Forscherinnen als Autorinnen aus der semantisch aufgeladenen und sentimentalisierten Konfiguration der körperlichen Berührung von Affe und Frau eine Kernszene wissenschaftlicher und methodischer Erkenntnis gewinnen. In den darauffolgenden Kapiteln soll diese Methodik und Epistemologie näher ins Auge gefasst werden. Die primatologischen Autorinnen entwickeln zwar eine neue affektbasierte Methodik in der Feldforschung, sie können damit aber an zumindest ein bereits bestehendes Modell aus der Ästhetik anknüpfen, welches Emotionalität epistemologisch nutzbar macht: das Gefühl des Erhabenen (Abschn. 2.3). Es zeigt sich, dass eine derartige Rolle des Gefühls im Feldforschungsaufenthalt - als Erkenntnis und Selbsterkenntnis - nicht allein ein Phänomen der ,Trimates' ist, sondern sich auch in Vorgängern (George Schaller) und Nachfolgern (Robert Sapolsky) findet. Zudem erfährt das Gefühl des Erhabenen in den Beschreibungen der Primatologie zugleich eine Problematisierung. In der Beschäftigung mit den Gorilla-Passagen verschiedener primatologischer Forschungsmemoiren lässt sich nachvollziehen, wie der Gorilla als Tier des Erhabenen bzw. als Auslöser des Gefühls des Erhabenen kreiert wird und so als Topos des Erhabenen fungiert, der mittlerweile als Konsumware abgerufen werden kann, wie sich kritisch reflektiert in der Literatur - hier am Beispiel von Lukas Bärfuss' Roman Hundert Tage - zeigt. Im vierten (Abschn. 2.4) stehen dann affektive Nahformen der Anerkennung im Zentrum, wie sie das gesellschaftliche und individuelle Zusammenleben gestalten, aber eben auch für die Etablierung und die

${ }^{164}$ Vgl. Haraway: Primate Visions, S. 149 ff. 
teilnehmende Beobachtung der Feldforschung als grundlegend betrachtet werden müssen: als Untersuchungsgegenstand und Differenzkriterium bei Jane Goodall, als - im Sinne der Anerkennungstheorie beschreibbares - Instrument der Etablierung einer Forschungsbeziehung und Stiftung von Solidarität mit dem Forschungstier bei Dian Fossey, und als Teil der Forscherinnenrolle im Feld und Medium narrativer Empathie bei Biruté Galdikas. Abschließend wird anhand zweier fiktionaler Bearbeitungen des Affe-Mensch-Spezieskontaktes aufgezeigt, inwiefern Fiktion als Experimentalraum für primatologische Emotionalität dienen kann (Abschn. 2.5): Während der Spielfilm Gorillas in the Mist (1988) verdeutlicht, wie eine tendenziöse Lesart der Forschungsmemoiren Emotionalität aus dem Forschungskontext in Richtung sentimentalen Selbsterlebens am Affen rückt, lässt sich Peter Goldsworthys Roman Wish (1995) als genuines Gedankenexperiment dazu betrachten, was die Konsequenzen eines intimen affektiven Forschungsverhältnisses und der durch dieses zutage geförderten Erkenntnisse über Affen als Subjekte sein könnten.

Der zweite Analyse-Teil richtet den Blick von den epistemologischen Fragen nach den Emotionen der Forschung zu ästhetischen Fragen nach der Form der Emotionalität im Text. Er widmet sich somit einer Affektpoetik der Forschungsmemoiren. Geht eine Affektpoetik mit Burkhardt Meyer-Sickendiek davon aus, „daß sich spezielle literarische Gattungen als von den menschlichen Affekten geprägte und von den Affekten erzählende Formen begreifen lassen", 165 so ist dieser Begriff treffend für das Anliegen der Arbeit, die anders als ein Teil der literaturwissenschaftlichen Emotionsforschung nicht nach der Rezeptionsästhetik von Texten fragt, sondern unterschiedliche Formen in den untersuchten Texten als Indikatoren von emotionalen Erfahrungen liest. ${ }^{166} \mathrm{Im}$ Laufe der Langzeitforschung mit Affen ,in situ' wenden sich die Beobachtungen nach den im ersten Teil der Arbeit aufgenommenen, überwiegend positiven emotionalen Phänomenen (Erstkontakt, das Gefühl des Erhabenen, Liebe, Zuneigung, Anerkennung, Vertrauen) nun den negativen Erlebnissen zu, die aus der Einsicht in die ,dunklen Seiten' des Primatischen entstehen. Dies stellt die Autor/innen angesichts eingeübter Affektlogiken vor affektive Herausforderungen, die sich, so die These, als textuelle Oberflächen-Irritation, d. h. an Genre- bzw. Textformauffälligkeiten erkennen lassen. Denn Genre, so verstehe ich es mit der Forschung, ${ }^{167}$ lässt sich

\footnotetext{
${ }^{165}$ Meyer-Sickendiek, Burkhard: Affektpoetik. Eine Kulturgeschichte literarischer Emotionen. Würzburg 2005, S. 9.

${ }^{166}$ Die Affektpoetik unterscheidet sich darin auch von der Affektlehre der Rhetorik. Es geht ihr nicht um die Modulierung von in Zuhörer/innen (ggf. Leser/innen) ausgelösten Affekten. „Vielmehr geht sie im produktions- und werkästhetischen Sinne der Frage nach, inwiefern die den literarischen Text prägenden affektuellen Regungen für eine literarische Gattung konstitutiv sind“. Meyer-Sickendiek: Affektpoetik, S. 9.

${ }^{167}$ Siehe Fowler, Alastair: Kinds of Literature. An Introduction to the Theory of Genres and Modes. Oxford 1982, S. 31; Devitt, Amy J.: Generalizing about Genre: New Conceptions of an Old Concept. In: College Composition and Communication 44/4 (1993), S. 573-586; auch Langer, Susanne K.: Feeling and Form. A Theory of Art. New York 1953; Frye, Northrop: Anatomy of Criticism. Four Essays. Princeton 1957, S. $223 \mathrm{ff}$.
} 
als ästhetischer Verarbeitungsprozess von Erfahrungen lesen; in diesem Kontext als Verfahren der Einordnung von abjekten Erfahrungen in das Narrativ der Feldforschung. ${ }^{168}$

Vorab kann hierzu eine Lektüre von Edgar Rice Burroughs' Abenteuerroman Tarzan of the Apes (1912/1914) als primatologische Fallstudie eines psychopathischen Übermenschen(affen) illustrierend herangezogen werden (Abschn. 3.1). Dass die Frage der Form wichtig für die Untersuchung der Affekte im Feld ist, zeigt sich dann daran, wie deutlich die Forschungsmemoiren auf verschiedene Genres und Modi zurückgreifen, um ambivalente Erfahrungen aus der Langzeitforschung zur Darstellung zu bringen. In (Abschn. 3.2) wird anhand des melodramatischen Modus und seines Einsatzes bei der Thematisierung primatischer Sexualität diskutiert, welche Funktion die Wahl von Textformen für das Registrieren, Verarbeiten, Einordnen und Darstellen herausfordernder Szenen tierlichen Verhaltens hat. Der melodramatische Modus bietet sich mit seinem festen Platz im kulturellen Repertoire ästhetischer Formen an, um dialektisch moralische Problemstellungen zu bearbeiten, die von den Forscher/innen Gefühlsarbeit ${ }^{169}$ als Arbeit an den eigenen Gefühlen im Abgleich mit dem jeweiligen Regularium objektiver Forschung - erfordern. Zugleich lässt er tierliches Verhalten mittels der Formalisierung in stereotypen Motiven, Figuren und Gesten auch für ein nicht-wissenschaftliches Publikum lesbar werden. Anhand der Einkehr extremer Gewaltbeobachtungen in die Texte der Langzeitforschung der Primatologie lässt sich im Anschluss (Abschn. 3.3) untersuchen, wie der Einsatz rhetorischer Mittel und die Wahl bestimmter literarischer Formen als ordnende Kategorisierungsstrategien für das zuvor Undenkbare dienen: Anhand der Textformen bzw. Genres, ihrer poetologischen Charakteristika und Implikationen findet eine Normalisierung oder Pathologisierung des problematischen beobachteten Gewaltverhaltens statt. Diese Kategorisierung dient letztlich auch dem Umgang mit den traumatischen Aspekten der Gewaltbeobachtung in der Primatologie. Tierliches Sterben und tierlicher Tod wiederum provozieren sowohl private wie auch politische Formen der Verlust-Verarbeitung im Text. Der Verlust von Forschungstieren ist in der Langzeitforschung so zwangsläufig wie schwerwiegend. Angesichts des individuellen tierlichen Todes und des drohenden Aussterbens der untersuchten Arten formieren sich in den Forschungsmemoiren zwei so literarische wie politische Genres, die sich mit jeweils einer der freudschen Formen des Umgangs mit Verlust identifizieren lassen (Abschn. 3.4): Der normativen Trauer als erfolgreicher Verarbeitung des Verlustes entspricht der Nachruf auf das Tier, der zum einen der schmerzhaften Emotionalität legitim Raum geben kann, zum anderen aber die

\footnotetext{
${ }^{168}$ Siehe dazu auch White, Hayden: Tropics of Discourse: Essays in Cultural Criticism. Baltimore 1978, S. 110: „the generic story-type serves as a conceptual model to which the events are to be likened in order to permit their encodation as elements of a recognizable structure", Hervorheb. im Original.

${ }^{169}$ Siehe Hochschild, Arlie Russell: Emotion Work, Feeling Rules, and Social Structure. In: American Journal of Sociology 85/3 (1979), S. 551-575.
} 
politische Implikation besitzt, das Tier als Individuum betrauerbar zu machen. Aus der Zerstörung der Umwelt erwächst die melancholische primatologische Klage, die ganz im Sinne einer Rhetorik der Artenelegie ${ }^{170}$ nicht nur anklagt, sondern auch zum Handeln aufrufen soll. Im abschließenden Abschn. 3.5 dieses Teils zur Affektpoetik der primatologischen Forschungsmemoiren wird am Beispiel von William Boyds Roman Brazzaville Beach (1990) gezeigt, dass sich Fiktion nicht nur damit auseinandersetzt, wie affektinduzierend Affen und Affenforschung sein können, sondern auch, welches Potenzial Affekte als Agenten von Veränderung besitzen. Aufgrund ihrer persönlichkeitsstrukturierenden Funktion müssen sie, so adaptiert Boyd Aristoteles' Affektlehre, in ein rechtes Maß gerückt werden. Der Roman führt derart unmäßige und mäßige Affektivität in ihren Facetten kathartisch auf, um zu verdeutlichen, was ein Ideal von Forschungsemotionalität sein könnte. Fiktion fungiert somit nicht nur als Resonanzraum für primatologische Emotionalität, sondern elaboriert eigenständig emotionstheoretische Ansätze und produziert in ihrer Qualität als ,Aufführungsort' emotionaler Vielfalt einen kathartischen Raum für ihre Ausdrucksformen.

Damit verweist dieses Zwischenabschlusskapitel auch bereits auf den dritten und letzten Untersuchungsteil der Arbeit, der sich den Affektregimen der Primatologie widmet und danach fragt, was die Erfahrungs- und Äußerungsbedingungen von Emotionalität für die Primatologie sind: $\mathrm{Ob}$ und wie Forschungsmemoiren und andere primatologische Monographien Emotionalität thematisieren, ist von den Emotionsregimen und Gefühlsregeln der Wissenschaft und den ideologischen Strukturen der sie einbettenden Gesellschaft abhängig. Als Auftakt (Abschn. 4.1) soll daher untersucht werden, wie zwei experimentell arbeitende frühe Primatologen, der Gestaltpsychologe Wolfgang Köhler und der Zoologe Solly Zuckerman, am Vorabend der Etablierung des physiologischen Behaviorismus von ihren Beobachtungen am Affen berichten. Dies bietet den Raum, sich anhand der historischen Pejoration von Anthropomorphismus und Ankedote mit diesen beiden epistemischen Phänomenen und dem an sie gebunden Vorwurf der Gefühligkeit bzw. des Sentimentalismus, kurz der Unwissenschaftlichkeit auseinanderzusetzen. Dabei lässt sich die jeweilige Strategie der Abgrenzung von beidem als taktischer Zug im Sinne der wissenschaftlichen Autoritätsgenerierung herausarbeiten. Daran anschließend (Abschn. 4.2) rückt die auf die Epoche der ,Trimates" folgende Generation von Forschungsberichten in den Blick. Diese Texte entstehen im neuen Forschungsparadigma der Soziobiologie, das auf einer Übernahme neueren ökonomischen Denkens in der Biologie bzw. einer Biologisierung der Ökonomie als Verhaltensökonomie beruht. Emotionen werden nun zunächst auf der Ebene der Forschung, aber auch auf der ihres Gegenstands, der Tiere, ausgespart. Durch die Ökonomisierung ihres Verhaltens werden Affen dabei zu strategisch handelnden Akteur/innen. Mit der Konsolidierung des Neoliberalismus als zugrunde liegender ökonomischer Schule werden Emotionen jedoch als Ressource wiederentdeckt.

\footnotetext{
${ }^{170}$ Siehe Heise, Ursula: Nach der Natur. Das Artensterben und die moderne Kultur. Frankfurt a. M. 2010, S. 73.
} 
Tiere gewinnen dadurch Emotionen als Motivationen wieder, während Emotionen selbst als Ressource für die Forschung aufbereitet werden. Ein anderes Beispiel für die Abhängigkeit der Darstellung und Thematisierung von Emotionalität in den Forschungsmemoiren zeigt sich an Robert Sapolskys Forschungsmemoiren (Abschn. 4.3). A Primate's Memoir lässt sich vor dem Hintergrund der Ironie-Diskussion um 2001 verstehen, in der zugleich ein Ende der postmodernen Ironie als Dekonstruktion von Ernsthaftigkeit und Verantwortung gefordert und zugleich der Wert der Ironie als (auch affektives) Instrument der (politischen) Kritik und als Ethos neu zutage gefördert wurde. Sapolsky, so meine These, nutzt Ironie als strategisches Verfahren der Immunisierung, das es ihm erlaubt, nicht nur Kritik an Forschungspraktiken und -ideologien zu üben, sondern auch emotionale Erfahrungen unterschiedlicher Ausprägung thematisieren zu können. Das nächste Kapitel (Abschn. 4.4) wird sich den beiden führenden deutschen Primatolog/innen widmen. Deren jüngste populärwissenschaftliche primatologische Texte lenken den Blick von der englischsprachigen auf die deutsche Literatur der Primatologie. An Volker Sommers Forschungsmemoiren Schimpansenland (2008) und Julia Fischers mit Anleihen an das Genre bestückter Monographie Affengesellschaft (2012) lässt sich die Übernahme der populärwissenschaftlichen Tradition der englischsprachigen Primatologie im Dienste einer Popularisierung des Forschungsgebietes aufzeigen. Diese Texte unterscheiden sich von der älteren englischsprachigen Literatur jedoch stilistisch, im Affektvokabular und im Erzählen von der Forschung bspw. durch Auffälligkeiten des anekdotischen Erzählens (Fischer) oder dezidiert technische Ausführungen und abwegige Mentalitätsdiskurse (Sommer). Die Leitthese hier ist, dass die veränderten Forschungsbedingungen im 21. Jahrhundert (weniger Affen, größerer Kenntnisstand, andere Methoden und Praktiken in der Feldforschung) nicht nur zu einer veränderten Affektivität im Feld führen, sondern auch zu veränderten Darstellungsweisen und Erzählelementen. Das fühlende Selbst des Forschungsberichts, das der Erzählung immer auch Authentizität zu verleihen versprach, muss daher stärker als bislang gestützt und supplementiert werden - teilweise im Rückgriff auf problematische ältere Genres wie den Afrikareisebericht. Um diesen Untersuchungsteil abzuschließen, wende ich mich dann mit zwei deutschen Romanen dem Bereich der emotionalen Ethik zu (Abschn. 4.5). Ulrike Draesners Sieben Sprünge vom Rand der Welt (2014) und Bettina Suleimans Auswilderung (2014) setzen Ethiken des Emotionalen in den Fokus ihrer Auseinandersetzung mit der primatologischen Forschung. Bei Draesner betrifft dies transgenerationale Traumata und individuelle affektive Persönlichkeitsprofile, die sich auf historische Gefühlskulturen und Erfahrungen zurückführen lassen, und die Frage nach der Rolle von Empathie für die epistemischen Möglichkeiten menschlichen Denkens und Verstehens. Bei Suleiman steht mit einer problematischen Erzählerinnenfigur zur Diskussion, welche Aufmerksamkeit ein gelungener Forschungsethos dem Emotionalen widmen muss: Der Roman wirft Fragen nach dem Selbstoptimierungsgebot unserer Gegenwart, nach akademischen Gefühlskulturen und Strukturen und danach auf, was eine zeitgenössische Tierethik für die Forschung mit Affen bedeuten wird. Die 
beiden Romane bieten somit einen fiktionalen Verhandlungsraum für reale Fragen zu einer Ethik des Emotionalen in der (primatologischen) Forschung.

Das Fazit der Arbeit wird schließlich einen kurzen Ausblick auf das aktuell in der Populärkultur spürbare Ergebnis der Emotionsarbeit der ,Science/Fiction * wagen und ihre Konsequenz für das Verhältnis von Affe, Mensch und Affekt andeuten.

Open Access Dieses Kapitel wird unter der Creative Commons Namensnennung 4.0 International Lizenz (http://creativecommons.org/licenses/by/4.0/deed.de) veröffentlicht, welche die Nutzung, Vervielfältigung, Bearbeitung, Verbreitung und Wiedergabe in jeglichem Medium und Format erlaubt, sofern Sie den/die ursprünglichen Autor(en) und die Quelle ordnungsgemäß nennen, einen Link zur Creative Commons Lizenz beifügen und angeben, ob Änderungen vorgenommen wurden.

Die in diesem Kapitel enthaltenen Bilder und sonstiges Drittmaterial unterliegen ebenfalls der genannten Creative Commons Lizenz, sofern sich aus der Abbildungslegende nichts anderes ergibt. Sofern das betreffende Material nicht unter der genannten Creative Commons Lizenz steht und die betreffende Handlung nicht nach gesetzlichen Vorschriften erlaubt ist, ist für die oben aufgeführten Weiterverwendungen des Materials die Einwilligung des jeweiligen Rechteinhabers einzuholen. 